ARTICLE

DOI: $10.1038 / s 41467-018-05660-0$

\title{
MYC dephosphorylation by the PP1/PNUTS phosphatase complex regulates chromatin binding and protein stability
}

Dharmendra Dingar ${ }^{1}$, William B. Tu1,2, Diana Resetca1,2, Corey Lourenco ${ }^{1,2}$, Aaliya Tamachi', Jason De Melo (D) 1, Kathleen E. Houlahan (10) 2,3, Manpreet Kalkat ${ }^{1,2}$, Pak-Kei Chan ${ }^{1}$, Paul C. Boutros (D) 2,3,4, Brian Raught ${ }^{1,2} \&$ Linda Z. Penn ${ }^{1,2}$

The c-MYC (MYC) oncoprotein is deregulated in over 50\% of cancers, yet regulatory mechanisms controlling MYC remain unclear. To this end, we interrogated the MYC interactome using BiolD mass spectrometry (MS) and identified PP1 (protein phosphatase 1) and its regulatory subunit PNUTS (protein phosphatase-1 nuclear-targeting subunit) as MYC interactors. We demonstrate that endogenous MYC and PNUTS interact across multiple cell types and that they co-occupy MYC target gene promoters. Inhibiting PP1 by RNAi or pharmacological inhibition results in MYC hyperphosphorylation at multiple serine and threonine residues, leading to a decrease in MYC protein levels due to proteasomal degradation through the canonical SCFFBXW7 pathway. MYC hyperphosphorylation can be rescued specifically with exogenous PP1, but not other phosphatases. Hyperphosphorylated MYC retained interaction with its transcriptional partner MAX, but binding to chromatin is significantly compromised. Our work demonstrates that PP1/PNUTS stabilizes chromatin-bound MYC in proliferating cells.

\footnotetext{
${ }^{1}$ Princess Margaret Cancer Centre, University Health Network, Toronto M5G $1 \mathrm{~L} 7$ ON, Canada. ${ }^{2}$ Department of Medical Biophysics, University of Toronto, Toronto M5G 1L7, Canada. ${ }^{3}$ Ontario Institute for Cancer Research, Toronto ON Canada M5G OA3, Canada. ${ }^{4}$ Department of Pharmacology and Toxicology, University of Toronto, Toronto, Canada M5S 1A8, Canada. Correspondence and requests for materials should be addressed to L.Z.P. (email: Ipenn@uhnres.utoronto.ca)
} 
n non-transformed cells, MYC protein expression is highly regulated by both transcriptional and post-transcriptional mechanisms, but MYC expression is deregulated in the majority of cancers. Deregulation occurs by well-established mechanisms involving gross genetic abnormalities (e.g., gene amplification or translocations) or by less defined mechanisms that can involve activated signaling cascades constitutively deregulating MYC activity ${ }^{1-4}$. MYC is a potent oncogene, in part because it is a master regulator that integrates multiple signaling cascades to regulate a wide variety of biological activities, including cellular proliferation, apoptosis, metabolism, and differentiation ${ }^{4-7}$. MYC orchestrates these activities by modulating gene transcription in association with MAX. The MYC-MAX heterodimer binds to E-box and non-E-box containing regulatory regions of $10-15 \%$ of all mammalian genes to control their expression and in turn, various biological processes ${ }^{8-10}$. MYC is highly responsive to signaling cascades, in part because it is a short half-life protein $(\sim 30 \mathrm{~min})$, that is primarily regulated by the well-characterized GSK3/SCFFBXW7 pathway. Mitogen regulated kinases phosphorylate MYC at serine 62 (Ser62). GSK3 $\beta$ then phosphorylates threonine 58 ( $\mathrm{Thr} 58$ ), which triggers protein phosphatase 2A (PP2A)-mediated Ser62 dephosphorylation. This ultimately leads to $\mathrm{SCF}^{\mathrm{FBXW7}}$ E3 ligase-mediated MYC ubiquitylation and subsequent proteasomal degradation ${ }^{1,11}$.

Evidence from mouse models show that targeting MYC preferentially triggers tumor cells to undergo differentiation and/or apoptosis, leading to tumor regression ${ }^{6,12,13}$. Developing MYC inhibitors would significantly benefit cancer patient care and outcome, yet targeting MYC directly using traditional approaches has not been successful ${ }^{14,15}$. More recently, inhibitors such as JQ1, targeting a bromodomain protein (BRD4), were shown to down-regulate expression of several genes important for tumor maintenance, including $\mathrm{MYC}^{16,17}$. Indeed, clinical grade BRD4 inhibitors have been fast-tracked to phase I clinical trials in a wide variety of malignancies in which MYC plays a role ${ }^{18}$. This paradigm of targeting essential MYC regulators is promising and suggests that building an arsenal of MYC inhibitors at multiple levels of regulation will increase efficacy through use in combination therapy.

To this end, our goal was to better understand the posttranslational modifications (PTMs) and regulators of MYC by interrogating the MYC interactome using BioID. We describe here the interaction of MYC with the PP1/PNUTS holoenzyme protein complex. MYC can induce PNUTS expression, suggesting a feed-forward co-operative regulatory loop. This is further supported by the co-localization of MYC and PNUTS to the promoters of MYC target genes. Inhibition of PP1/PNUTS triggers hyperphosphorylation of MYC, leading to chromatin eviction and degradation by the canonical $\mathrm{SCF}^{\mathrm{FBXW} 7}$ pathway. PP1/PNUTS is amplified in multiple cancer types, suggesting a model in which elevated PP1/PNUTS expression confers a growth advantage by increasing MYC protein stability.

\section{Results}

MYC BioID identifies the PP1/PNUTS heterodimeric complex. To investigate the regulation of MYC beyond the level of transcription, we evaluated PTMs and protein interactors of MYC. To assess MYC PTMs in a MYC-dependent transformation system, we used MCF10A cells. This is a genomically stable, nontransformed breast epithelial cell line that becomes transformed in response to ectopic deregulated MYC expression ${ }^{19}$. To determine whether MYC was post-translationally modified, we established a 2D electrophoresis assay in which MYC was immunoprecipitated from MCF10A cells, then separated by 2D electrophoresis, and immunoblotted. Interestingly, MYC migrated as several distinct "spots" suggesting that MYC harbors numerous PTMs in these growing cells (Fig. 1a). These several distinct MYC spots could be the result of many PTMs, including phosphorylation, acetylation, methylation, and/or glycosylation.

To identify MYC regulators, we used a proximity-dependent biotinylation technique, BioID ${ }^{20,21}$. Many of the technical problems associated with identifying MYC interactors using traditional approaches ${ }^{22}$ have been overcome with our recent development of MYC-BioID ${ }^{20}$. The N-terminus of full length MYC was fused in-frame with a BirA R118G (BirA*), an E. coli mutant protein biotin ligase. Ectopic expression of FLAGBirA*MYC in cells supplemented with exogenous biotin allows proteins that are in close proximity to FLAGBirA*-MYC to be biotinylated. These biotinylated proteins are then enriched by affinity purification using streptavidin and identified by mass spectrometry (MS). This technique significantly increases the sensitivity and specificity of the detection of MYC interactors compared to previous MS based methods ${ }^{20,22}$. We generated HeLa cells to express FLAGBirA* control or FLAGBirA*-MYC and performed MYC-BioID as described ${ }^{20}$. Consistent with our previously identified MYC protein interactome conducted in HEK293 cells grown in tissue culture or as a tumor xenograft ${ }^{20}$, we identified many known (e.g., MAX, TRRAP, WDR5) ${ }^{23-25}$ and novel protein interactors using BioID (Fig. 1b; Supplementary Table 1).

Given that MYC is highly post-translationally modified (Fig. 1a), we prioritized our analysis on novel enzymatic interactors that were identified using MYC-BioID (Fig. 1b). To better understand phosphorylation-mediated regulation of MYC $1,19,26-30$, we focused on several novel MYC interactors that together form the PP1/PNUTS phosphatase complex, including the protein phosphatase 1 catalytic subunit alpha (PP1 $\alpha$; gene name: $P P P 1 C A$ ), protein phosphatase 1 catalytic subunit gamma (PP1 $\gamma$; gene name: PPP1CC), and protein phosphatase-1 nucleartargeting subunit (PNUTS; gene name: PPP1R10) (Fig. 1c). PP1 is a member of the PPP family of Ser/Thr phosphatases. Their substrate specificity, activity, and subcellular localization are controlled by interactions with regulatory subunits. The PP1 catalytic subunit has three isoforms $(\alpha, \beta$, and $\gamma$ ), which interacts with a number of regulatory subunits and works as a heterodimeric holoenzyme ${ }^{31}$. PNUTS is a well characterized regulatory subunit of PP1 and targets PP1 to its substrates ${ }^{32,33}$. Using MYC-BioID, we identified the PP1/PNUTS enzyme complex as a potential novel regulator of MYC.

MYC and PP1/PNUTS are amplified in cancer. To determine whether MYC and its putative interactor, PP1/PNUTS, are both expressed in cancer, we examine the amplification status of the $M Y C$ gene, as well as genes of the PP1/PNUTS holoenzyme (PPP1CA, PPP1CB, PPP1CC, and PPP1R10) in The Cancer Genome Atlas (TCGA) datasets. Significant co-amplification for MYC and genes encoding the PP1/PNUTS complex was evident in patient tumor samples of several solid cancer types that severely impact human health, including breast (Fig. 2a), lung (adeno and squamous), and uterine carcinomas (Supplementary Fig. 1a-c). This suggests that the PP1/PNUTS phosphatase complex may contribute to the regulation of MYC in cancer, providing relevance for further investigation.

MYC has been previously shown to induce expression of its regulators, as part of a feed-forward regulatory loop ${ }^{34}$. To determine whether MYC can induce PP1/PNUTS expression, we evaluated whether MYC is bound to these gene regulatory regions. First, we analyzed ENCODE MYC and MAX ChIP-seq datasets, which revealed that MYC and MAX are both bound to the PPP1R10, PPP1CA, PPP1CB, and PPP1CC gene promoters 
a
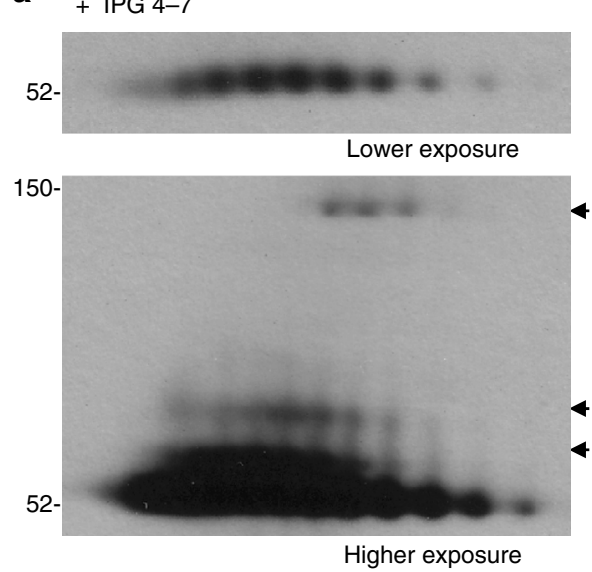

IP: Anti-MYC mouse antibody

IB: Anti-MYC rabbit antibody
C

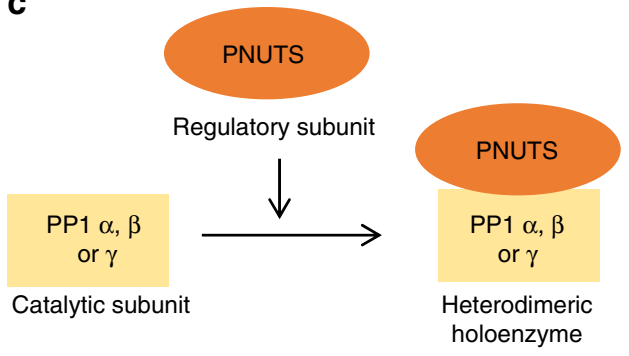

b

\begin{tabular}{|c|c|c|c|c|c|c|c|c|c|c|c|c|}
\hline & & \multicolumn{5}{|c|}{ FLAGBirA* control } & \multicolumn{6}{|c|}{ FLAGBirA*-MYC } \\
\hline Gene name & & \multicolumn{2}{|c|}{ Pool A } & \multicolumn{2}{|c|}{ Pool B } & Total & \multicolumn{2}{|c|}{ Pool A } & \multicolumn{2}{|c|}{ Pool B } & Total & SAINT \\
\hline$M Y C$ & & & & & & & 42 & 39 & 35 & 34 & 150 & \\
\hline MAX & & & & & & & 18 & 14 & 13 & 16 & 61 & 1 \\
\hline EP400 & & & & & & & 294 & 252 & 251 & 223 & 1020 & 1 \\
\hline TRRAP & $\xi$ & & & & & & 305 & 255 & 224 & 223 & 1007 & 1 \\
\hline$H D A C 2$ & है & 11 & 14 & 4 & 2 & 31 & 33 & 25 & 28 & 28 & 114 & 1 \\
\hline WDR5 & & & & & & & 8 & 6 & 6 & 7 & 27 & 1 \\
\hline SMARCA4 & & 5 & 5 & 3 & & 13 & 35 & 32 & 36 & 33 & 136 & 1 \\
\hline TAF6L & & & & & & & 4 & 2 & 2 & 2 & 10 & 0.99 \\
\hline PPP1R10 & & 19 & 7 & 12 & 6 & 44 & 57 & 40 & 34 & 37 & 168 & 0.99 \\
\hline PPP1CA & రి & & & & & & 4 & 2 & 3 & 7 & 16 & 0.96 \\
\hline PPP1CC & & & & & & & 2 & 9 & 2 & 2 & 15 & 1 \\
\hline
\end{tabular}

Fig. $1 \mathrm{MYC}$ is post-translationally modified and interacts with the PP1/PNUTS phosphatase complex. a Cell lysate from growing MCF10A cells was immunoprecipitated with MYC monoclonal mouse antibody, resolved by 2D gel electrophoresis ( $7 \mathrm{~cm}, \mathrm{pH} 4-7$ IPG strip; $10 \%$ SDS-PAGE), transferred onto nitrocellulose membrane and immunoblotted with MYC polyclonal rabbit antibody. Representative image of $n=3$. Shown is lower exposure (top panel) and higher exposure (bottom panel) x-ray film images. Arrows show numerous high molecular weight MYC spots. b Shown are the gene names and spectral counts of selected known and novel MYC interacting proteins identified by conducting MYC-BiolD MS in HeLa cells. A and B are biological replicates with two technical replicates shown for each. Total spectral counts and SAINT analysis score are presented. Significance is achieved with SAINT $>0.8$. c Schematics of protein phosphatase 1 (PP1) and protein phosphatase 1 nuclear-targeting subunit (PNUTS). The catalytic subunit of PP1 $\alpha, \beta$, or $\gamma$ interacts with a regulatory subunit such as PNUTS, which forms a heterodimeric holoenzyme that interacts with PP1:PNUTS substrates

(Supplementary Fig. 2). We also evaluated the expression of PNUTS in HO15.19 MYC knockout cells, with and without ectopic MYC expression and found that PNUTS protein expression is increased in response to ectopic MYC expression (Fig. 2b). Additionally, our own MYC ChIP analysis validated that MYC is bound to the PPP1R10 promoter, as well as a known MYC-induced gene $(C A D)$, but not to a negative control region (chr6) (Fig. 2c). Taken together, our data suggest MYC controls the expression of its interactors PP1/PNUTS through a feedforward regulatory loop.

MYC:PNUTS interaction is supported by PLA and ChIPqPCR. The regulatory subunit (PNUTS) is the substrate specifying factor for the catalytic PP1 subunit. Therefore, we validated the MYC-PNUTS interaction by proximity ligation assay (PLA) in MCF10A cells. Addition of the rabbit MYC- or mouse PNUTS-specific antibodies alone yielded only background levels of fluorescence in cells (Fig. 2d); however, a specific nuclear fluorescence signal was detected when cells were probed with both MYC and PNUTS antibodies (Fig. 2d images (above) and histogram (below)). To further evaluate MYC-PNUTS interaction beyond the MCF10A cell line, we also evaluated A549 (lung adenocarcinoma), Daudi (lymphoma) (Supplementary Fig 3a images (left) and histogram (right)), OCI-AML3 (AML3) (leukemia), and H520 (squamous cell lung cancer) (Supplementary Fig. 3b images (left) and histogram (right)) cells and observed MYC-PNUTS interaction. To validate the specificity of the MYC PLA signal, we also performed PLA in HO15.19 MYC knockout cells. The MYC-null cells did not produce any fluorescence signal, but when MYC expression was restored there was an increase in signal with the MYC-PNUTS antibody pair (Supplementary Fig. 4a), demonstrating that the PLA signal was specific to MYC. Additionally, we also tested the specificity of PNUTS antibody in HEK293 cells transiently transfected with PNUTS sgRNA. PNUTS antibody detected a single band at the expected molecular weight in empty vector transfected cells, but this band was not evident in PNUTS sgRNA cells (Supplementary Fig. 4b), indicating that the PNUTS antibody is specific to its target. Taken together these data suggest that endogenous MYC and PNUTS interact in intact cells (Fig. 2d), and this interaction can be 
a

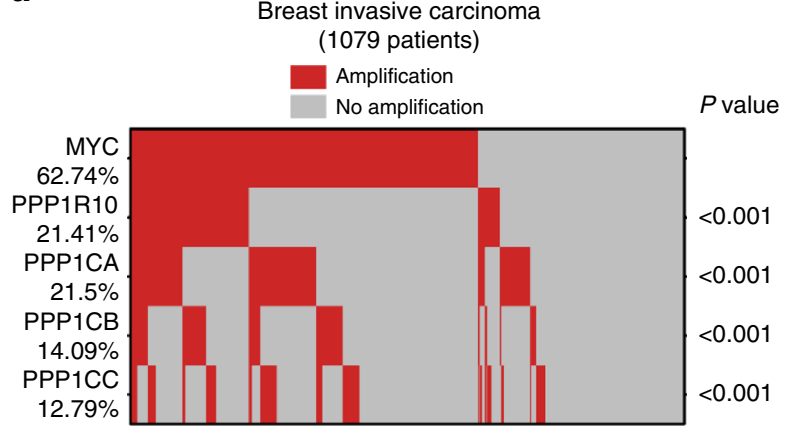

d
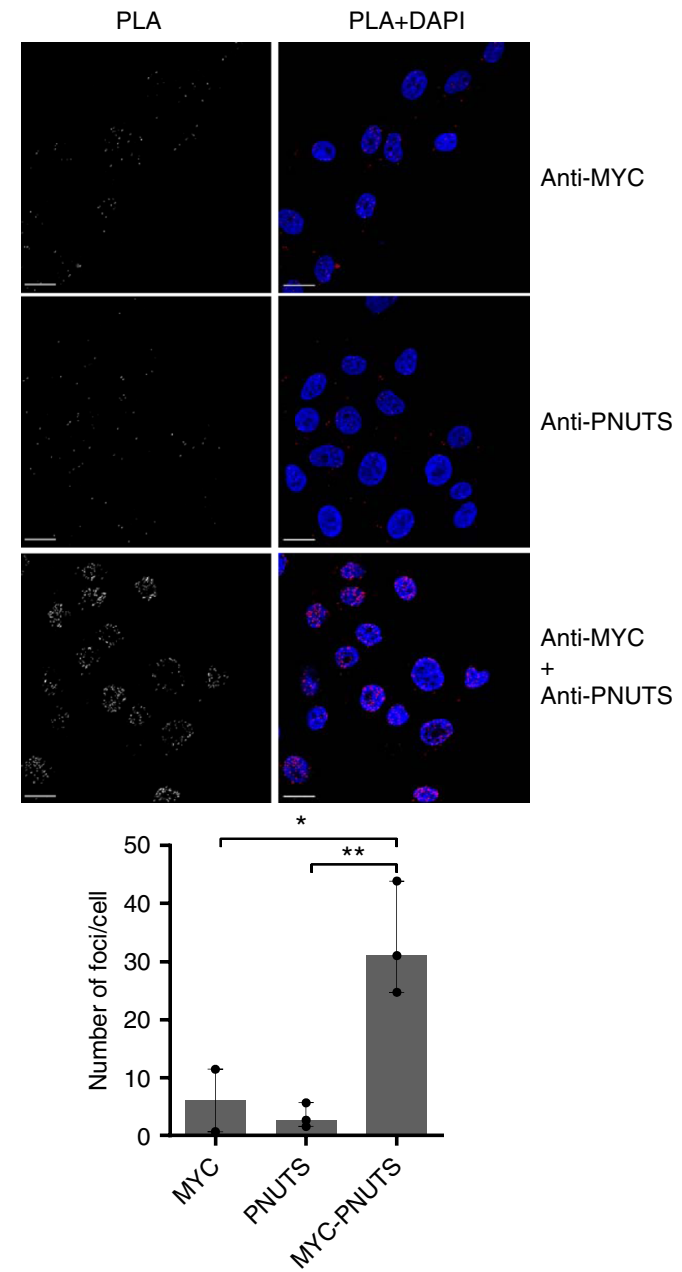

b

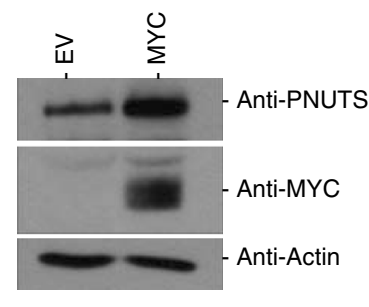

e

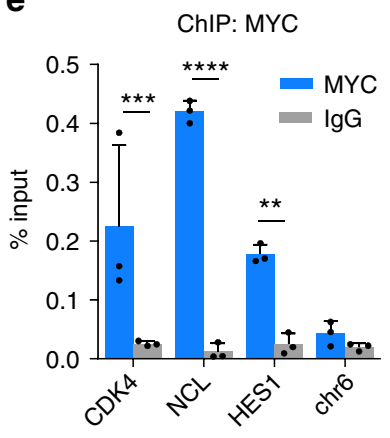

ChIP: PNUTS

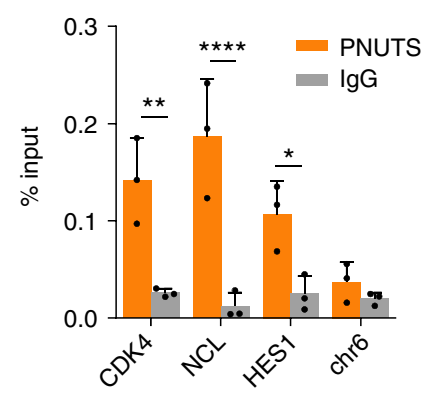

C

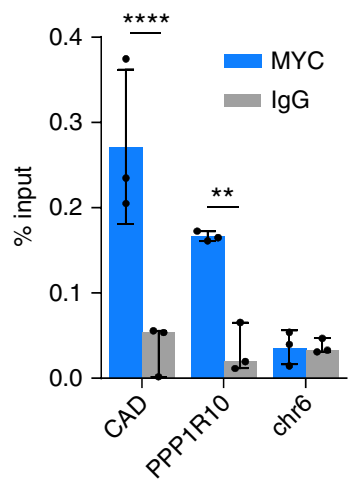

f

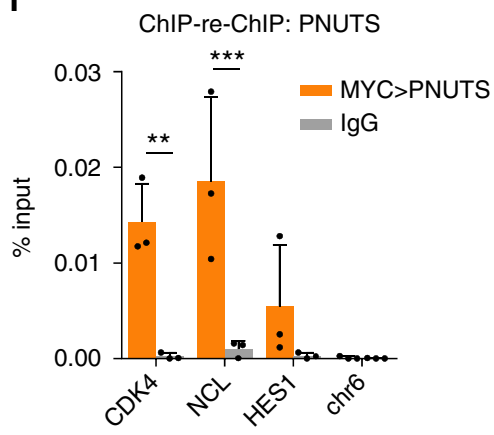

ChIP-re-ChIP: MAX

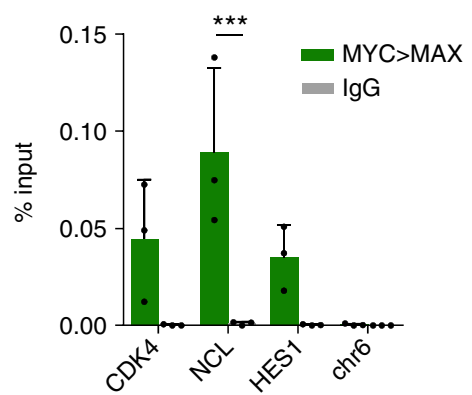

Fig. 2 MYC and PP1/PNUTS are co-amplified in cancer and co-bind to promoters of MYC target genes. a MYC and PPP1R10, PPP1CA, PPP1CB, or PPP1CC gene amplification is evident and demonstrates significant co-occurrence in breast invasive carcinoma. b HO15.19 MYC knockout cells (EV) or cells ectopically expressing MYC were lysed and immunoblotted with antibodies to PNUTS, MYC, and actin. Shown are representative images of $n=3$. c MCF10A cells were analyzed by chromatin immunoprecipitation (ChIP) using MYC or IgG antibody. ChIP'd DNA was quantified by qPCR for MYC or IgG binding to CAD and PPP1R10 promoters or chr6 (negative control). d MCF10A cells probed with MYC rabbit antibody and/or a PNUTS mouse antibody. The proximity of MYC and PNUTS was assayed using the Duolink Proximity Ligation Assay (PLA) In Situ Red Starter kit (Sigma) as per manufacturer's instructions. Representative PLA signal (red or white) and nuclear staining (DAPl; blue) are shown for cells probed with anti-MYC alone (top row), antiPNUTS alone (middle row), or anti-MYC and anti-PNUTS (bottom row). PLA signal was quantified and shown as median number of foci per cell with range $(n=3) .{ }^{\star} p<0.05,{ }^{\star \star} p<0.01$, one-way ANOVA with Bonferroni test. Scale bars represent $20 \mu \mathrm{m}$. e MCF10A cells analyzed by ChIP with MYC, PNUTS or IgG antibody. ChIP'd DNA was quantified using qPCR for MYC, PNUTS or IgG binding to MYC target genes promoters, CDK4, NCL, and HES1, or chr6 (negative control). f For ChIP-re-ChIP analysis, MCF10A cells lysate incubated with MYC rabbit antibody followed by PNUTS (top panel), or MAX (bottom panel) antibody along with IgG antibody as a control. ChIP'd DNA was quantified similarly as above for ChIP experiment. For c, e, f: shown are \% input mean $(n=3) .{ }^{\star} p<0.05,{ }^{\star \star} p<0.01,{ }^{\star \star \star} p<0.001,{ }^{\star \star \star \star} p<0.0001$, two-way ANOVA with Bonferroni test. Error bars represent s.d. 
detected in a variety of cell lines by PLA (Supplementary Fig. 3 and 4).

To test whether MYC and PNUTS bind to MYC target gene promoters, MYC or PNUTS was subjected to ChIP-qPCR with target specific antibodies in MCF10A cells. MYC (Fig. 2e, top) or PNUTS (Fig. 2e, bottom) both displayed significant enrichment at promoters of known MYC target genes: CDK4 (Cyclin Dependent Kinase 4), NCL (Nucleolin), and HES1 (Hes Family BHLH Transcription Factor 1) compared to IgG control. A chromosome 6 (chr6) non-E box containing region was used as negative control. To evaluate the MYC and PNUTS co-binding to MYC target gene promoters, we performed MYC ChIP-re-ChIP with PNUTS (Fig. 2f, top) or MAX as a positive control (Fig. 2f, bottom) antibody. Both ChIP-re-ChIP analyses showed enrichment of MYC-MAX and MYC-PNUTS on MYC target gene promoters. These data indicate that MYC and PNUTS are bound to promoters of the same set of MYC target genes.

MYC hyperphosphorylation and degradation. To explore the functional relevance of the MYC interaction with PP1/PNUTS, we inhibited PP1 using Calyculin A, a pharmacological PP1 inhibitor. MCF10A cells exposed to Calyculin A for $30 \mathrm{~min}$ were harvested and analyzed by immunoblot using an antibody to MYC-pT58 or MYC (Fig. 3a, left). In response to PP1 inhibition, multiple phospho-MYC species (pMYC), as indicated using antiMYC pT58, migrated at higher molecular weight (Fig. 3a, top blot) and the abundance of total MYC was reduced (Fig. 3a, middle blot). The decreased level of MYC protein was not due to reduction in total MYC mRNA (Fig. 3a, right), as MYC mRNA levels, by contrast, increased after Calyculin A treatment. This may be due to a homeostatic feedback response, in an attempt to rescue the decreased MYC protein levels. The reduction in total MYC protein abundance in response to Calyculin A was both dose-dependent and time-dependent (Fig. 3b). The hyperphosphorylated MYC species were captured and visualized only in response to a relatively acute exposure $(30 \mathrm{~min})$ (Fig. $3 \mathrm{~b}$, top) but not to a longer exposure ( $2 \mathrm{~h}$ ) (Fig. $3 \mathrm{~b}$, bottom) to Calyculin A. This suggests that MYC hyperphosphorylation occurs prior to degradation and does not accumulate in sufficient abundance for detection at the lower doses and longer time points of Calyculin A treatment due to resultant degradation of MYC (Fig. 3b). The cells did not appear to be undergoing apoptosis in response to Calyculin A as we did not observe PARP cleavage after a 30-min treatment (Supplementary Fig. 5a). MYC degradation in response to Calyculin A was blocked by treatment with the proteasomal inhibitor MG132, enabling the higher migrating hyperphosphorylated species of MYC to be readily visualized (Fig. 3c). To test the specificity of PP1 inhibition on MYC levels, two different siRNA cocktails targeting all PP1 catalytic subunits (PP1 $\alpha, \beta$, and $\gamma)$ were evaluated. Consistently, PP1 knockdown resulted in lower levels of MYC protein compared to control. MYC protein levels, but not PP1, could be partially rescued with 4-h MG132 treatment (Fig. 3d). PP1 knockdown did not significantly change MYC mRNA levels (Supplementary Fig. 5b).

To evaluate these results beyond MCF10A cells, we assayed the response to PP1 inhibition across a panel of cell lines. These include primary non-transformed, hTERT-immortalized human mammary epithelial cells (HMEC), breast cancer (MDA-MB-231, BT549, HCC1954, HCC1937, HS578T) (Fig. 3e), leukemia (OCIAML2, OCI-AML3, referred to as AML2 and AML3 respectively), lymphoma (Raji, Daudi), lung adenocarcinoma (A549, HCC827), squamous cell lung cancer (H520, H2170), and uterine corpus endometrial carcinoma (HEC-1-A) cell lines (Supplementary Fig. 5c). These different cells lines universally displayed a similar response to Calyculin A treatment, which demonstrates that PP1/
PNUTS regulation of MYC phosphorylation and degradation occurs across multiple cell types. As the Raji B-cell lymphoma cells carry a mutation of MYC T58 ${ }^{35}$, the pT58 signal was not detectable in these cells (Supplementary Fig. 5c). This data reinforces the specificity of MYC phospho antibody to pT58 and importantly also demonstrates the involvement of phospho residues in addition to T58 in the Calyculin A-induced MYC molecular weight shift.

Calyculin A inhibits both PP1 and PP2A ${ }^{36}$, therefore it was important to determine whether inhibition of PP2A showed the same effect on MYC stability as Calyculin A. To this end, we transduced MCF10A cells with recombinant adenovirus that expressed SV40 small T antigen, a specific inhibitor of $\mathrm{PP} 2 \mathrm{~A}^{37}$. Small $\mathrm{T}$ antigen increased Ser62 phosphorylation as expected and increased MYC levels (Fig. 3f), but did not result in MYC degradation as was seen with Calyculin A-dependent PP1 inhibition (Fig. 3a). Additionally, we also treated cells with Okadaic acid, a PP2A inhibitor, which increased Ser62 phosphorylation (Supplementary Fig. 5d), but did not result in MYC degradation as was seen with PP1 inhibition. Taken together, these data suggest that an increase in MYC phosphorylation at multiple sites triggers MYC degradation, and PP1 is required for MYC stability.

PP1 dephosphorylates MYC. We next evaluated whether exogenous PP1 or another phosphatase could remove Calyculin Ainduced hyperphosphorylation of MYC. Briefly, MYC was immunoprecipitated from lysates of growing MCF10A cells treated with or without Calyculin A. The immunoprecipitated material was treated with exogeneous PP1 or calf intestinal alkaline phosphatase (CIP), and then immunoblotted with MYC pT58 or MYC antibody (Fig. 4a). The results demonstrated that introduction of PP1, but not CIP, resulted in dephosphorylation of both basal and PP1 inhibitor-induced MYC phosphorylation in control and Calyculin A-treated cells, respectively, showing that exogenous PP1 could reverse the hyperphosphorylation of MYC in response to Calyculin A. The lack of CIP effect was not due to loss of its enzyme activity. We tested CIP and PP1 phosphatase activity at the end of the 2-h incubation period with $\mathrm{p}$ Nitrophenyl phosphate (PNP) as a substrate (Fig. 4a bottom). Indeed, CIP activity was several fold higher than PP1 but still CIP could not dephosphorylate MYC. This suggests that the slower migrating bands were indeed the result of accumulated phosphorylation of MYC and that PP1 can dephosphorylate MYC.

MYC degradation is primarily regulated by the $\mathrm{SCF}^{\mathrm{FBXW7}} \mathrm{E} 3$ ligase $^{1,11}$; however several additional E3 ligases have recently been shown to regulate MYC activity ${ }^{1}$. To determine the role of the canonical SCF FBXW7 pathway, we studied the effect of PP1 inhibition by Calyculin A in HCT116 FBXW7 knockout or wildtype cells; knockout cells carry the FBXW7 gene exon 5 deletion which encodes for an inactive enzyme ${ }^{38}$. These cells were virally transduced and selected to express empty vector, MYC wildtype or the MYC T58A mutant and treated with Calyculin A or vehicle control. Consistently, PP1 inhibition by Calyculin A treatment resulted in lower levels of MYC in FBXW7 wild-type cells compared to $F B X W 7$ knockout cells (Fig. 4b; compare lanes 7-12 to 1-6). The FBXW7 knockout status in HCT116 cells was validated by PCR (Supplementary Fig. 5e). MYC T58A levels were also decreased but not to the same extent as MYC wildtype, suggesting additional E3 ligases can contribute to Calyculin A induced degradation of MYC (Fig. 4b; compare lanes 10 and 12). Similarly, the important functional role of FBXW7 to MYC degradation following PP1 inhibition was also evident in these cells following siRNA knockdown of PP1 (Fig. 4c). This demonstrates that MYC degradation in response to PP1 
a

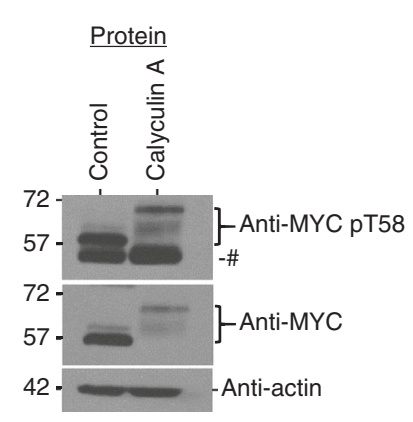

b

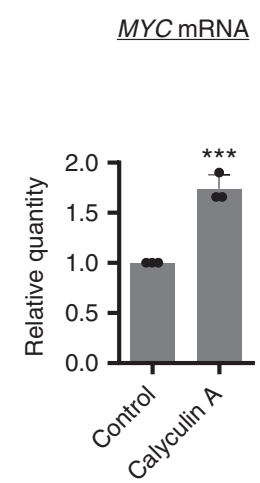

d

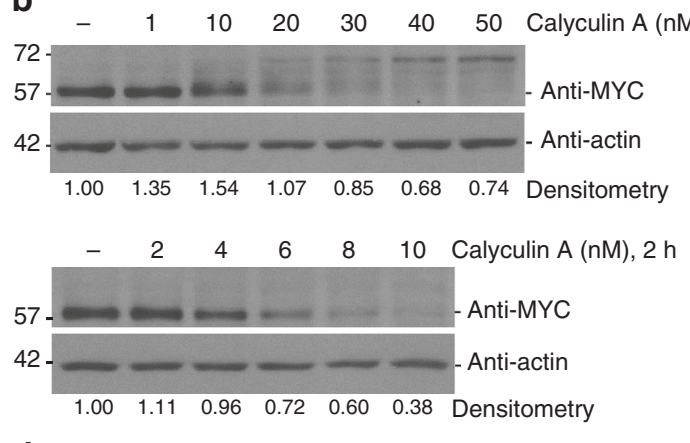

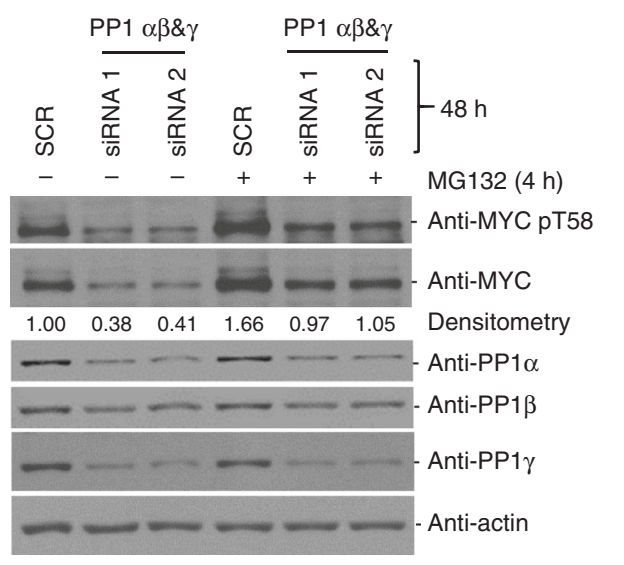

e

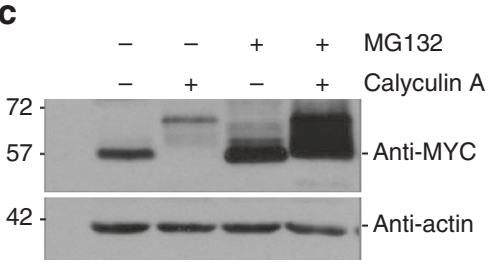

f

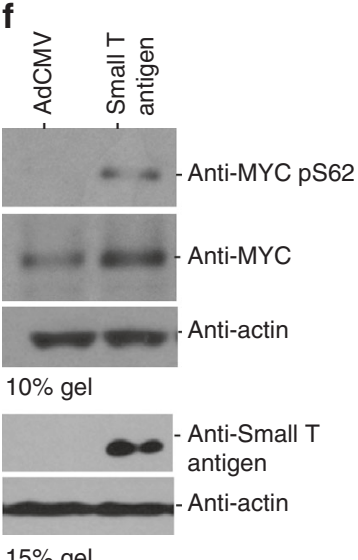

Fig. 3 Inhibition of PP1 results in decreased MYC protein levels. a MCF10A cells treated with Calyculin A (50 nM) for 30 min and lysed for protein or RNA extraction. Immunoblots of lysates were probed with MYC mouse, MYC pT58 rabbit, or actin rabbit antibody (left panel). \# indicates non-specific band. CDNA was made from extracted RNA and GRT-PCR was performed with primers against MYC or RPLPO as an internal control. Data are from three biological replicates, mean is shown. ${ }^{\star \star \star} p<0.001$; unpaired $t$-test, error bars represent s.d. b MCF10A cells were treated with Calyculin $A$ at $1,10,20,30$, 40, $50 \mathrm{nM}$ for $30 \mathrm{~min}$ (top panel) or 2, 4, 6, 8, $10 \mathrm{nM}$ for $2 \mathrm{~h}$ (bottom panel) and lysed. Immunoblots were probed with MYC mouse or actin rabbit antibody. c Growing MCF10A cells were treated with MG132 $(10 \mu \mathrm{M})$ for $4 \mathrm{~h} \pm$ Calyculin A (50 nM) for 30 min and lysed. Immunoblots were probed with MYC mouse or actin rabbit antibody. $\mathbf{d}$ Growing MCF10A cells transfected with $10 \mathrm{nM}$ siRNA directed against PP1 $\alpha, \beta$, and $\gamma$ or universal scramble negative control by calcium phosphate. After $44 \mathrm{~h}$ transfection, cells were treated with $10 \mu \mathrm{M}$ MG132 or vehicle control for $4 \mathrm{~h}$ and lysed. Immunoblots were probed with shown antibodies. e Growing HMEC, MDA-MB-231, BT549, HCC1954, HCC1937, or HS578T cells were treated with Calyculin A (50 nM) for 30 min and lysed. Immunoblots of lysates were probed with shown antibodies. $\mathbf{f}$ MCF10A cells expressing ectopic MYC were seeded, grown overnight, serum starved for $24 \mathrm{~h}$, and then infected with $200 \mathrm{MOI}$ of empty vector adenovirus (AdCMV) or adenovirus carrying small T antigen. Cells were grown for $22 \mathrm{~h}$ in $0.25 \%$ serum media, lysed, and lysates immunoblotted with indicated antibodies. Shown are representative images of $n=3$

inhibition is primarily due to the canonical $\mathrm{SCF}^{\mathrm{FBXW} 7}$ pathway with additional, minor contributions from other unknown mechanisms.

PP1 regulates several MYC serine/threonine residues. We next determined which of the many potential phosphorylation sites of
MYC were dephosphorylated by PP1. MCF10A cells were exposed to MG132 to block MYC degradation and then treated with vehicle control or Calyculin A (Fig. 3c). Equal amounts of MYC were immunoprecipitated, subjected to tryptic digestion followed by immobilized metal affinity chromatography (IMAC)based phosphopeptide enrichment, and phosphopeptides were identified by MS. Spectral counts for specific MYC 
a

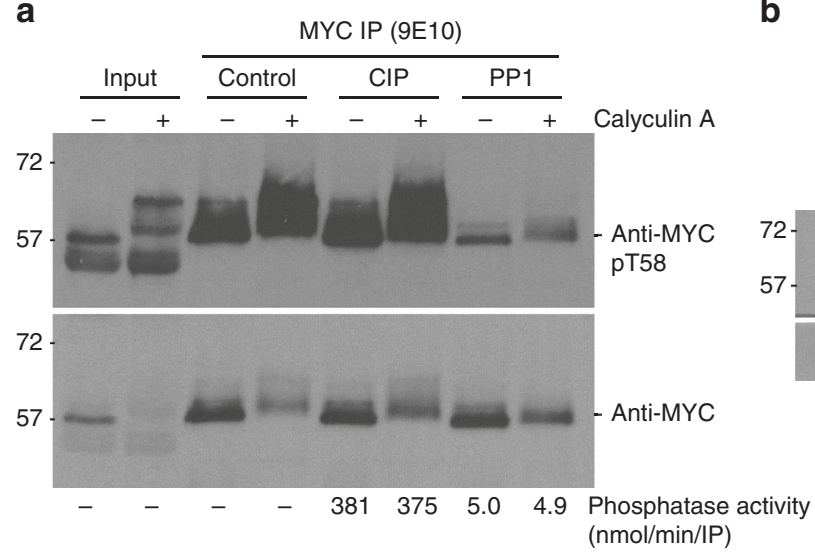

b

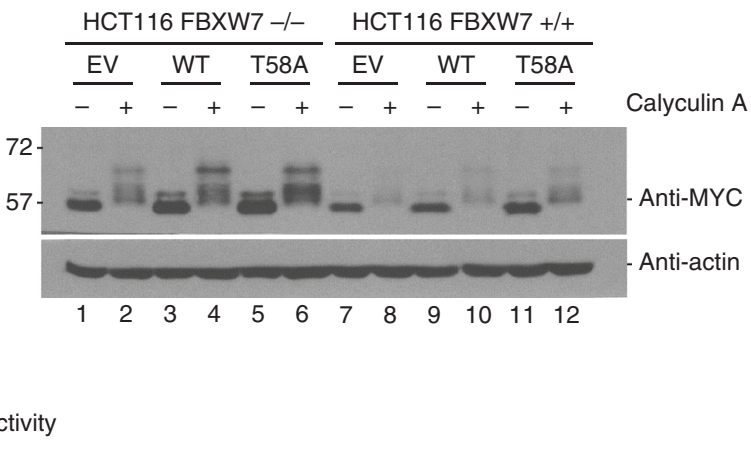

C
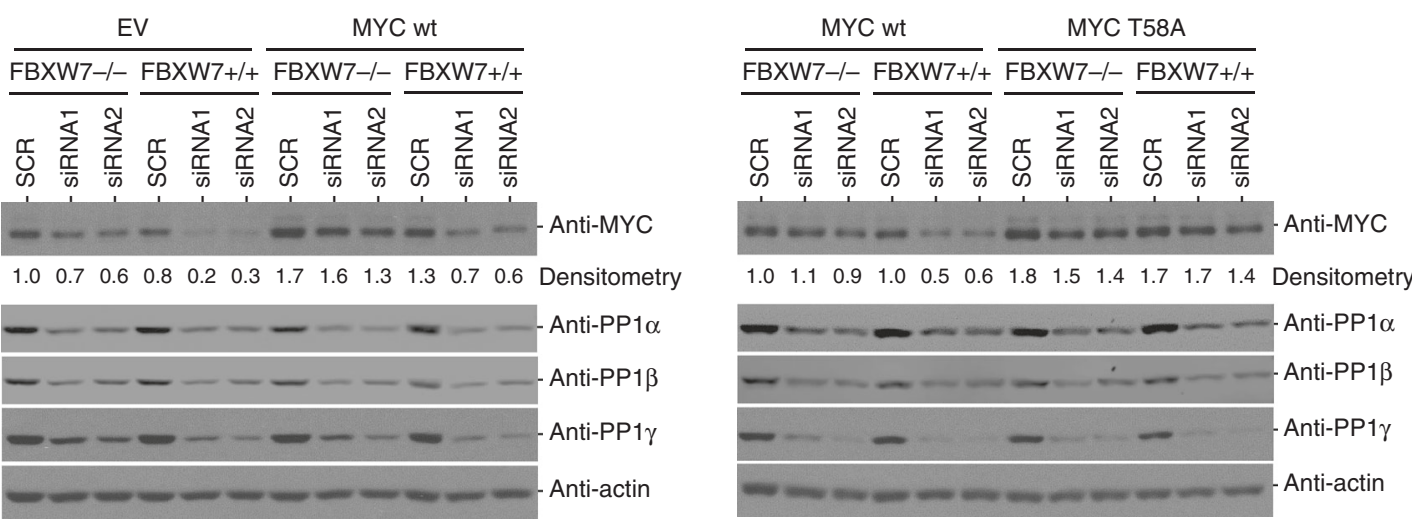

Fig. 4 PP1 dephosphorylates MYC and rescues it from SCFFBXW7-dependent degradation. a MCF10A cells were treated with Calyculin A (50 nM) or DMSO control for $30 \mathrm{~min}$, lysed, and immunoprecipitated with MYC mouse antibody. The IP was treated with an enzyme control, calf intestinal alkaline phosphatase (CIP) or protein phosphatase 1 (PP1) for $2 \mathrm{~h}$ at $30^{\circ} \mathrm{C}$. Total CIP or PP1 enzyme activity in the IP reaction after the 2-h incubation was measured using the substrate $p$-Nitrophenyl phosphate (PNP). The IP was loaded on a 10\% SDS-PAGE, transferred onto nitrocellulose membrane and immunoblotted with MYC pT58 rabbit or MYC rabbit antibody. Shown are representative images of $n=3$. b HCT116 FBXW7 knockout ( $-/-$ ) or wild-type $(+/+)$ cells expressing inducible ectopic empty vector (EV), wildtype MYC (wt) or MYC T58A (T58A) following 4-hydroxytamoxifen (4-OHT) (48 h) were treated with vehicle control $(-)$ or Calyculin $\mathrm{A}(50 \mathrm{nM})(+)$ for 30 min or $\mathbf{c}$ transfected with $10 \mathrm{nM}$ siRNA directed against PP1 $\alpha, \beta$, and $\gamma$ or universal scrambled negative control by calcium phosphate for $48 \mathrm{~h}$ and lysed. Protein lysates (20 $\mu \mathrm{g}$ ) were loaded onto 10\% SDS-PAGE, transferred onto nitrocellulose membrane and immunoblotted with indicated antibodies. Normalized MYC densitometry values are shown below the MYC blot. Shown are representative images of $n=3$

phosphopeptides were increased in response to Calyculin $\mathrm{A}$ treatment (Fig. 5a). Increased phosphorylation levels were observed at previously characterized (Thr58, Ser62, Ser71, Ser81, Ser344, Ser347, and Ser348) and novel phospho-residues (Ser151, Ser159, Ser161, Ser314, and Thr315). Thus, the phosphorylation of numerous residues was regulated by PP1, suggesting their potential role in regulating MYC degradation.

To evaluate the role of the PP1 modified residues identified by MS, we performed site directed mutagenesis of the Ser/Thr residues phosphorylated in response to PP1 inhibition. Interestingly, mutating these twelve Ser/Thr residues (Thr58, Ser62, Ser71, Ser81, Ser151, Ser159, Ser161, Ser314, Thr315, Ser344, Ser347, and Ser348) to Ala (MYC12A) inhibited the majority of the Calyculin A triggered shift and degradation (Fig. 5b). The lack of complete rescue suggests that additional residues not detected by MS may be modified in response to PP1 inhibition.

To evaluate the effect of these 12 phosphorylated residues on MYC stability, we evaluated the MYC wildtype and MYC12A protein half-life by treating cells with cycloheximide. Consistently, mutating these twelve Ser/Thr MYC residues resulted in an increased protein half-life from 27.84 to $57.04 \mathrm{~min}$ (Fig. 5c). Thus, after PP1 inhibition, MYC is phosphorylated on several Ser/Thr residues. Mutation of 12 of these residues to Ala precluded the majority of MYC hyperphosphorylation and degradation that were evident following PP1 inhibition, and increased MYC halflife.

MYC hyperphosphorylation reduces chromatin binding. As MYC interaction with MAX is required for MYC transcription ${ }^{3,9}$, we examined the ability of hyperphosphorylated MYC to interact with MAX. MAX was immunoprecipitated from MCF10A cells with or without Calyculin A exposure and immunoblotted for MYC (Fig. 6a). Hyperphosphorylated MYC and MAX interaction was intact, suggesting that MYC hyperphosphorylation does not interfere with MAX binding. To evaluate the ability of hyperphosphorylated MYC to bind chromatin, MCF10A cells were treated with MG132 and either vehicle control or Calyculin A, and then ChIP-qPCR was conducted using anti-MYC or IgG control to evaluate binding to MYC target gene promoters. MYC ChIP under hyperphosphorylated conditions showed significant reduction in MYC binding to MYC target genes, including $C A D$, CDK4, NCL, and LDHA gene promoters compared to MYC ChIP 
a

\begin{tabular}{|c|c|c|c|c|c|}
\hline Phospho species & \multicolumn{2}{|c|}{ MG132 } & \multicolumn{2}{|c|}{$\begin{array}{l}\text { Calyculin A+ } \\
\text { MG132 }\end{array}$} & Peptide sequence \\
\hline $\mathrm{T}[58]$ & 14 & 16 & 14 & 27 & \\
\hline$S[62]$ & 11 & 28 & 53 & 53 & ( \\
\hline $\mathrm{T}[58]$ and S [62] & 79 & 73 & 181 & 184 & (K) KFELLPT ${ }^{\mathbf{5 8}}$ PPL $\boldsymbol{S}^{6 \mathbf{2}} \mathrm{PSR}(\mathrm{R})$ \\
\hline$S[71]$ or $S[73]$ & 10 & 6 & 20 & 19 & \\
\hline$S[81]$ & & & 4 & 4 & (P) \\
\hline S [71] and S [81] & & 1 & & 2 & (R) SGLC $\boldsymbol{S}^{71}$ PS $^{73}$ YVAVTPF $\boldsymbol{S}^{81} L R(G)$ \\
\hline$S[151]$ & & & 1 & 1 & (K) LA $\boldsymbol{S}^{151}$ YQAAR $(K)$ \\
\hline$S$ [159] or S [161] & 8 & 13 & 13 & 20 & (P) \\
\hline$S$ [159] and S [161] & & & 4 & 3 & (K) $\mathrm{DS}^{159} \mathrm{GS}^{161} \operatorname{PNPAR}(\mathrm{G})$ \\
\hline$S[314]$ & 3 & 4 & 20 & 18 & (Р \\
\hline$S$ [314] or T [315] & 1 & 1 & 31 & 36 & (R) CHVSTHQHNYAAP PS ${ }^{\mathbf{3 1 4}} \mathrm{T}^{\mathbf{3 1 5}} \mathrm{R}(\mathrm{K})$ \\
\hline S [344] and S [347] or S [348] & 8 & 12 & 48 & 43 & DTEENVK (R) \\
\hline
\end{tabular}

b

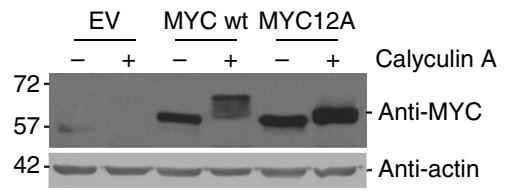

C
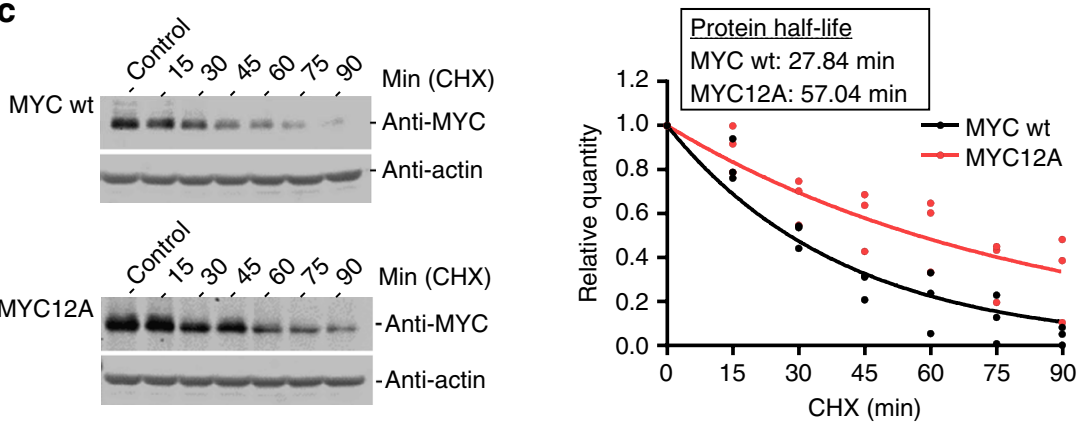

Fig. 5 PP1 dephosphorylates multiple serine and threonine residues on MYC. a Growing MCF10A cells were treated with MG132 (10 $\mu$ M) for $4 \mathrm{~h} \pm$ Calyculin A $(50 \mathrm{nM})$ for $30 \mathrm{~min}$, lysed, and MYC immunoprecipitated and then subjected to trypsin digestion, phospho-enrichment and analysis by MS. Shown are the peptide counts from two technical replicates as well as the peptide with the indicated phospho-sites. b MCF10A cells treated with 4-OHT (48 h) to induce expression of empty vector (EV) wildtype MYC (MYC wt) or a MYC mutant in which the 12 Ser and Thr residues identified in a were mutated to Ala (MYC12A), were treated with Calyculin A $(50 \mathrm{nM})$ for $30 \mathrm{~min}$ and lysed. Protein lysate $(20 \mu \mathrm{g})$ was loaded on 10\% SDS-PAGE, transferred onto nitrocellulose membrane and immunoblotted with MYC mouse or actin rabbit antibody. Shown are representative images of $n=3$. c MCF10A cells expressing MYC wt or MYC12A after 4-OHT $(24 \mathrm{~h})$ induction were treated with cycloheximide $(\mathrm{CHX})(10 \mu \mathrm{g} / \mathrm{mL})$, harvested at $15-\mathrm{min}$ intervals, lysed, resolved by SDS-PAGE and immunoblots probed with MYC or actin antibody (left). The MYC band intensities were quantified and signal normalized to actin. Shown are the normalized MYC signals for three replicates with protein half-life determinations (right)

under control conditions (Fig. 6b). By contrast, PNUTS binding to these MYC target gene promoters was unaffected under similar conditions (Supplementary Fig. 6a). IgG control ChIP showed little difference between the two conditions on any of the MYC target genes. Additionally, to test chromatin integrity and control for any global effects of Calyculin A, we performed ChIP-qPCR analysis using a Histone $\mathrm{H} 3$ antibody and observed no significant differences in chromatin integrity (Supplementary Fig. 6b). To further evaluate the subcellular localization of hyperphosphorylated MYC, nuclear-cytoplasmic fractionation was performed in MCF10A cells treated with MG132 and either vehicle control or Calyculin A (Fig. 6c left), or transfected with PP1 siRNA (Fig. $6 \mathrm{c}$ right). Irrespective of Calyculin A treatment or PP1 knockdown, MYC pT58 or total MYC was predominantly detected in nuclear fractions.

To further evaluate the effect of MYC hyperphosphorylation on chromatin binding by an alternative approach, we mutated the
12 Ser/Thr MYC phospho-residues to either Ala (MYC12A) or the phosphomimetic Asp (MYC12D) and introduced inducible expression vectors carrying EV, N-terminally FLAG-tagged MYC or these MYC mutants into MCF10A cells. Cells induced with 4OHT and MG132 treatment showed comparable FLAG-tagged MYC wildtype, MYC12A, or MYC12D expression (Fig. 6d top). However, ChIP with anti-FLAG or IgG control showed significant reduction in MYC12D binding to MYC target genes, including $C A D, C D K 4, L D H A$, and NCL gene promoters, compared to MYC wildtype or MYC12A (Fig. 6d below). This suggests that the phosphomimetic MYC12D mutant mimics MYC phosphorylation on these $12 \mathrm{Ser} / \mathrm{Thr}$ residues and antagonizes chromatin binding. In summary, MYC hyperphosphorylation on twelve Ser/Thr residues has no effect on MAX binding, but significantly alters MYC binding to target gene promoters and demonstrates a novel post-translational mechanism to control MYC activity. 
a

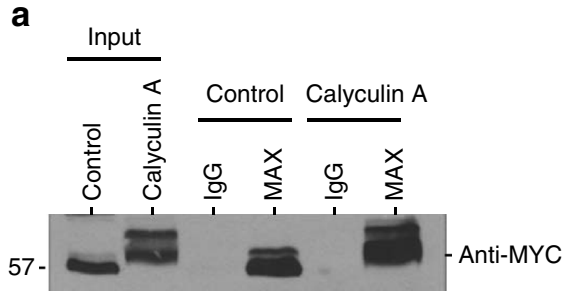

\section{C}

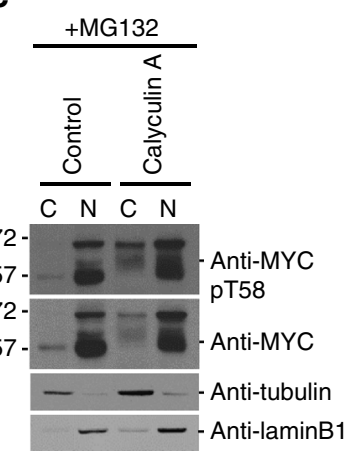

b

$$
\begin{array}{cc}
\frac{\text { ChIP: MYC }}{\text { DMSO }} & \frac{\text { ChIP: IgG }}{\text { DMSO }} \\
\text { Calyculin A } & \text { Calyculin A }
\end{array}
$$

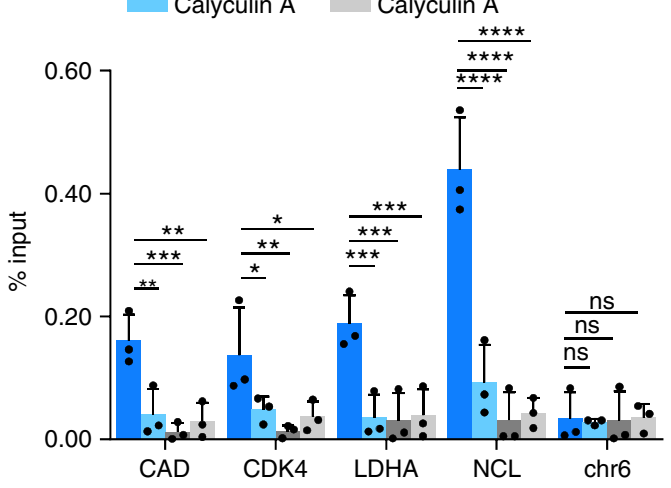

d

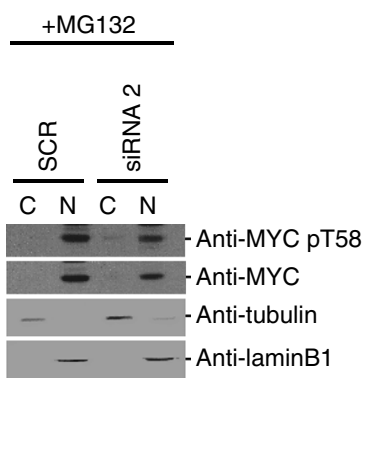

e
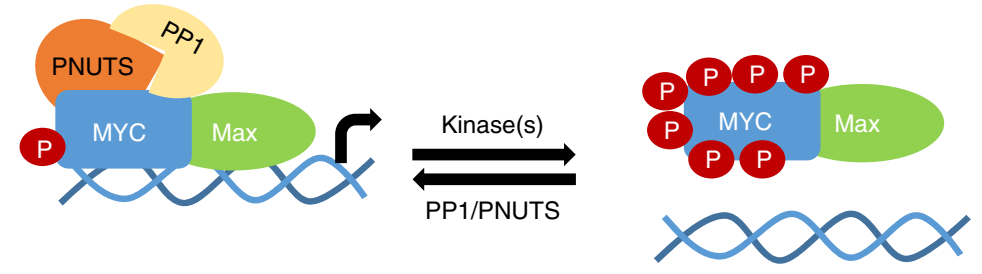

FBXW7 (Ub) ${ }^{\mathrm{n}}$ or other E3

ligase $(\mathrm{Ub})^{\mathrm{n}}$

USPS
Anti-FLAG

- Anti-actin

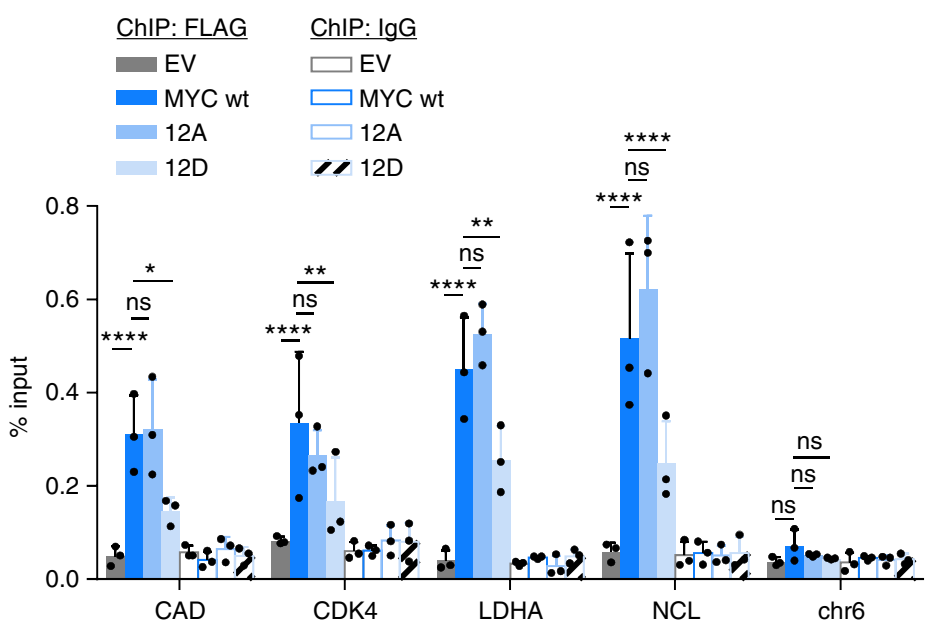

Fig. 6 Hyperphosphorylated MYC retains interaction with MAX but is compromised for chromatin binding. a MCF10A cells were treated with Calyculin A $(50 \mathrm{nM})$ or vehicle control for $30 \mathrm{~min}$, lysed, and immunoprecipitated with MAX rabbit or lgG rabbit antibody. The input and IP was resolved by SDS-PAGE and immunoblotted with MYC mouse antibody. Shown is a representative image of $n=3$. $\mathbf{b}$ For ChIP analysis, MCF10A cells were treated with MG132 (10 $\mu \mathrm{M})$ for $4 \mathrm{~h} \pm$ Calyculin $\mathrm{A}(50 \mathrm{nM})$ for $30 \mathrm{~min}$, and lysate incubated with MYC rabbit antibody along with IgG antibody as a control. ChIP'd DNA was quantified by qPCR for MYC target genes promoters of CAD, CDK4, LDHA, and NCL, and chr6 (negative control). c For nuclear and cytoplasmic fractionation, MCF10A cells were treated with MG132 $(10 \mu \mathrm{M})$ for $4 \mathrm{~h} \pm$ Calyculin $\mathrm{A}(50 \mathrm{nM})$ for 30 min (left panel), or transfected with $10 \mathrm{nM}$ siRNA directed against PP1 $\alpha, \beta$, and $\gamma$ or universal scramble negative control by calcium phosphate for $44 \mathrm{~h}$ and then treated with $\mathrm{MG} 132$ (10 $\mu \mathrm{M}$ ) for $4 \mathrm{~h}$ (right panel). Lysates were used for fractionation, and immunoblotted with indicated antibodies. d MCF10A cells carrying inducible FLAG-tagged EV, MYC wt, MYC12A, or MYC12D were treated with 4-OHT (24h) to induce expression, treated with MG132 (10 $\mu \mathrm{M})$ for $4 \mathrm{~h}$, and then lysed for immunoblot analysis (top) or for ChIP-qPCR with FLAG or IgG antibody (bottom) as outlined in $\mathbf{b}$ above. For $\mathbf{b}$, $\mathbf{d}$ : shown are $\%$ input mean (MYC and FLAG ChIP: $n=3$ ). ${ }^{*} p<$ $0.05,{ }^{\star \star} p<0.01,{ }^{\star \star \star} p<0.001,{ }^{\star \star \star \star} p<0.0001$, two-way ANOVA with Bonferroni test. Not statistically significant $=$ ns. Error bars represent s.d. e Proposed model of PP1/PNUTS regulation of MYC hyperphosphorylation in the control of chromatin binding and degradation 


\section{Discussion}

We conducted an unbiased MYC-BioID MS protein-protein interaction screen and identified the PP1/PNUTS holoenzyme as a MYC interactor. PLA was used to validate the endogenous MYC-PNUTS interaction and sequential ChIP-re-ChIP of MYC followed-by PNUTS revealed that both proteins bind to MYCbound gene promoters. MYC can induce PP1/PNUTS expression, suggesting a feed-forward loop in which MYC drives PP1/PNUTS expression to enable their interaction and regulate gene transcription. Consistent with these results, recent genome-wide DamID analysis demonstrated that PP1 and PNUTS promoterbinding significantly overlaps with MYC ChIP-seq datasets from the ENCODE consortium ${ }^{39}$. This is consistent with our ChIP results and suggests that MYC and PP1/PNUTS promoter occupancy can co-occur genome-wide. RNAi and pharmacological inhibitors of PP1 trigger the hyperphosphorylation of MYC, resulting in chromatin dissociation and MYC protein degradation through the canonical SCF ${ }^{\mathrm{FBXW7}}$ pathway. Importantly, exogenous introduction of PP1 can rescue MYC from this hyperphosphorylated state. PP1/PNUTS genes are co-amplified in several cancer types, suggesting that one role of this phosphatase is to dephosphorylate MYC to keep it in a stable, chromatinbound configuration.

At first glance, it is somewhat surprising that MYC activity and stability are enhanced by PP1/PNUTS, considering MYC is one of the mitogen-stimulated, immediate early genes whose expression is routinely up-regulated in response to a wide-variety of progrowth stimuli ${ }^{2}$. It was anticipated that these mitogen-activated kinase signaling cascades would lead to MYC phosphorylation, which would promote MYC activity. However, our data in conjunction with the literature paint a different picture in which phosphorylation can both promote and restrict MYC activity. The specific residues that are modified and the degree of phosphorylation appear to determine the net effect on MYC function. For example, phosphorylation of MYC Ser62 by MAPK increases MYC activity, but also primes Thr58 for phosphorylation, which promotes MYC ubiquitylation and degradation ${ }^{1}$. This phosphorylation is highly regulated as a recent study showed that Thr58 phosphorylation could be removed by the EYA1 phosphatase $^{40}$. Mutating MYC Thr58 to Ala resulted in increased MYC stability and transformation ${ }^{19}$ and mutation of this site is evident in Burkitt Lymphoma ${ }^{41}$, thus locking MYC into a more stable and active form. Concomitant regulation of multiple phosphorylation sites can also impact MYC stability and/or activity. For example, we have previously shown that mutating Ser71 or Ser81 to Ala individually had no effect on MYC dependent transformation, but mutating both S71/S81 or another cluster of MYC phospho-sites, Thr343/Ser344/Ser347/Ser348, to Ala increased the ability of MYC to drive transformation ${ }^{19}$. MYC phosphorylation on Thr358, Ser373, and Thr400 by the stressresponsive kinase Pak2 reduces MYC activity by interfering with MAX dimerization or DNA binding ${ }^{28}$. MYC phosphorylation on Ser373 by PKC $\zeta$ reduces MYC activity ${ }^{42}$ and phosphorylation on Thr244 results in reduced stability ${ }^{29}$. In the mitotic phase of the cell cycle, MYC hyperphosphorylation has been suggested to reduce MYC binding to chromatin during chromatin condensation $^{43}$. Thus, MYC regulation by site-specific phosphorylation involves single or multiple, often clustered, residues and primarily leads to reduced activity and/or stability.

Based on previous studies and our results, we propose a simple model: when MYC is bound to chromatin and regulating gene transcription, it resides in a relatively less phosphorylated state. Upon hyperphosphorylation, MYC no longer binds chromatin and is then either dephosphorylated by PP1/PNUTS and recycled to regulate gene transcription or is degraded through the SCF ${ }^{\text {FBXW7 }}$ or other E3 ligase mediated pathways (Fig. 6e). These multiple phosphorylation events may lead to a change in overall charge that may then alter MYC interaction with proteins and/or DNA. Thus, phosphorylation of MYC can negatively regulate MYC activity.

The detailed interaction of PP1/PNUTS with most of its substrates remains unclear. Interestingly, RNA polymerase II (RNAPII) has been established as an endogenous substrate for the PP1/PNUTS holoenzyme to regulate the dephosphorylation of the C-terminal domain at residue Ser $5^{33,44}$. Given that MYC has been shown to play a role in both the initiation and elongation of gene transcription, it is tempting to speculate that PP1/PNUTS may regulate both MYC and RNAPII to control gene transcription. Further studies to better define the molecular basis of PP1/ PNUTS with MYC in the context of transcription control will provide insight as to how PP1/PNUTS regulates MYC phosphorylation and function. This information will also guide the design of inhibitors targeting the PP1/PNUTS and MYC interaction to drive the inactivation and degradation of MYC.

\section{Methods}

Cell lines. MCF10A cells were cultured as previously described ${ }^{45}, \mathrm{HO} 15.19$ (kindly provided by Dr. John Sedivy), MDA-MB-231 (HTB-26), HS 578T (HTB-126), HCT116 (CCL-247), and HeLa (CCL-2) cells were cultured in DMEM supplemented with 10\% FBS, HCC1937 (CRL-2336), BT549 (HTB-122), HCC1954 (CRL 2338), A549 (CRM-CCL-185), HCC827 (CRL-2868), Raji (CCL-86, kindly provided by Dr. Eleanor Fish), H520 (HTB-182, kindly provided by Dr. Christine Allen), H2170 (CRL-5928), and Daudi (CCL-213) cells were cultured in RPMI1640 supplemented with 10\% FBS, OCI-AML2 (ACC-99) and OCI-AML3 (ACC582) cells were cultured in Alpha-MEM supplemented with 10\% FBS, HEC-1-A cells (HTB-112) were cultured in McCoy's 5A supplemented with $10 \%$ FBS, and HMEC (CRL-3243) cells were cultured in MEGM with BPE, hEGF, hydrocortisone, GA-1000, and insulin (Lonza CC-3150). HCT116 FBXW7-/- or $+/+$ cells were obtained from Dr. Lars-Gunner Larson with permission from Dr. Bert Vogelstein.

BiolD in cell culture. HeLa cells at $\sim 70 \%$ confluency, carrying tetracyclineinducible FLAGBirA* or FLAGBirA*-MYC, were treated for $24 \mathrm{~h}$ with $1 \mu \mathrm{g} / \mathrm{mL}$ tetracycline, $5 \mu \mathrm{M}$ MG132, and $50 \mu \mathrm{M}$ biotin, to induce the expression of the transgene and facilitate biotin labelling. Cells were then scraped into phosphate buffered saline (PBS), washed two times with $25 \mathrm{~mL}$ of PBS, and centrifuged at $1000 \times g$ for $5 \mathrm{~min}$ at $4{ }^{\circ} \mathrm{C}$. Cell pellets were lysed in $10 \mathrm{~mL}$ of cold modified RIPA buffer (w:v; 1\% NP-40, $50 \mathrm{mM}$ Tris-HCl pH 7.5, $150 \mathrm{mM} \mathrm{NaCl}, 1 \mathrm{mM}$ EDTA, 1 mM EGTA, 0.1\% SDS, 1:500 protease inhibitor cocktail (Sigma) 0.5\% sodium deoxycholate), supplemented with $250 \mathrm{U}$ of benzonase (EMD). Lysate was endover-end rotated for $1 \mathrm{~h}$ at $4^{\circ} \mathrm{C}$, sonicated $3 \times 30 \mathrm{~s}$ (Fisher Scientific D100 Sonic Dismembrator), and centrifuged at $27,000 \times g$ for $30 \mathrm{~min}$ at $4^{\circ} \mathrm{C}$. Biotinylated proteins were isolated by affinity purification with $30 \mu \mathrm{g}$ of (RIPA-equilibrated) streptavidin-sepharose beads (GE) with end-over-end rotation for $2 \mathrm{~h}$ at $4{ }^{\circ} \mathrm{C}$. Beads were washed $7 \times 1 \mathrm{~mL} 50 \mathrm{mM}$ ammonium bicarbonate $(\mathrm{pH} 8.0)$ prior to tryptic digest.

Mass spectrometry for BiolD. Tryptic digestion was performed with $1 \mu \mathrm{g}$ of MS grade TPCK trypsin (Promega, Madison, WI) dissolved in $100 \mu \mathrm{L}$ of $50 \mathrm{mM}$ ammonium bicarbonate ( $\mathrm{pH} 8.0$ ) which was added to the streptavidin-sepharose beads and incubated at $37^{\circ} \mathrm{C}$ overnight. The eluate was collected and beads were washed twice in $150 \mu \mathrm{L}$ of $50 \mathrm{mM}$ ammonium bicarbonate. Eluate and washes were pooled, lyophilized and reconstituted in $0.1 \%$ formic acid. Liquid chromatography analysis was performed on an in-house analytical column $(75 \mu \mathrm{m}$ inner diameter $)$ and pre-column $(150 \mu \mathrm{m}$ inner diameter $)$, made from fused silica capillary tubing from InnovaQuartz (Phoenix, AZ), and packed with $100 \AA$ C18-coated silica particles (Magic, Michrom Bioresources, Auburn, CA).

Peptides were resolved and identified using reversed phase $(120 \mathrm{~min}$ buffer gradient $10-40 \%$ acetonitrile, $0.1 \%$ formic acid) nanoflow liquid chromatographyelectrospray ionization-tandem mass spectrometry (nLC-ESI-MS/MS), running at $250 \mathrm{~nL} / \mathrm{min}$ on a Proxeon EASY-nLC pump in-line with a hybrid linear quadrupole ion trap Orbitrap mass spectrometer, Velos LTQ (ThermoFisher Scientific, Waltham, MA). A parent ion scan was performed in the Orbitrap using resolving power of 60,000. Up to 20 most intense peaks (minimum ion count of 1000) were selected for MS/MS using standard CID fragmentation. Fragment ions were detected in the LTQ. Dynamic exclusion was activated, where MS/MS of the same $\mathrm{m} / z$ (within a $10 \mathrm{ppm}$ window, exclusion list size 500) detected two times within 15 $s$ were excluded from analysis for $30 \mathrm{~s}$. For protein identification, Proteowizard ${ }^{46}$ was used to convert Thermo.RAW files to the.mzXML, and then searched using X! Tandem ${ }^{47}$ against Human RefSeq Version 45 (appended with cRAP and reversed decoy database based on RefSeq v45). Search parameters specified a parent MS 
tolerance of $15 \mathrm{ppm}$ and an MS/MS fragment ion tolerance of $0.4 \mathrm{Da}$, with up to two missed cleavages allowed for trypsin. Oxidation of methionine and ubiquitylation of lysine residues were allowed as variable modifications. Data were analyzed using trans-proteomic pipeline ${ }^{48}$ via the ProHits software suite ${ }^{49}$. Proteins identified with ProteinProphet cut-off of 0.8 (corresponding to FDR $<1 \%$ ) were analyzed with SAINT Express v. 3.350,51

Mass spectrometry for PTMs. PTM analysis of MYC was performed in MCF10A cells. Briefly, $2 \times 10^{8}$ MCF10A cells were grown to approximately $60 \%$ confluency and treated with $10 \mu \mathrm{M}$ of MG132 for $4 \mathrm{~h}$ or with $10 \mu \mathrm{M}$ MG132 for $4 \mathrm{~h}$ and $50 \mathrm{nM}$ Calyculin A for $30 \mathrm{~min}$. Cell were then scraped into PBS, pooled and washed once with $50 \mathrm{~mL}$ of PBS with centrifugation at $1000 \times g$ for $5 \mathrm{~min}$ at $4{ }^{\circ} \mathrm{C}$. Cell pellets were lysed in $1 \mathrm{~mL}$ of modified Cell Lysis Buffer (w:v; $1 \%$ SDS, $100 \mathrm{mM}$ Tris-HCl $\mathrm{pH} 6.8,1 \mathrm{x}$ protease inhibitor cocktail (Sigma)), supplemented with $250 \mathrm{U}$ of benzonase (EMD), $250 \mu \mathrm{M}$ sodium orthovanadate, $10 \mathrm{mM} \mathrm{NaF}$ and $50 \mathrm{mM}$ betaglycerolphosphate, $\mathrm{pH} 7.5$. Lysed cells were then collected into a $50 \mathrm{~mL}$ Falcon tube, boiled for $10 \mathrm{~min}$ at $95^{\circ} \mathrm{C}$ and sonicated $3 \times 30 \mathrm{~s}$. Solution was reconstituted to 20 $\mathrm{mL}$ with PBS and centrifuged at $27,000 \times \mathrm{g}$ for $30 \mathrm{~min}$ at $4{ }^{\circ} \mathrm{C}$. Cell lysates were then used for MYC IP using $60 \mu \mathrm{L}$ commercially crosslinked $9 \mathrm{E} 10$ antibody beads (Sigma). Samples were incubated overnight at $4{ }^{\circ} \mathrm{C}$. Beads were washed $7 \times 1 \mathrm{~mL}$ of $50 \mathrm{mM}$ ammonium bicarbonate ( $\mathrm{pH}$ 8.0). MYC protein was eluted off the beads in two steps using $300 \mu \mathrm{L}$ of MS Elution Buffer (0.5 M Ammonium hydroxide) Eluates were pooled and lyophilized. Samples were resuspended in $200 \mu \mathrm{L}$ of 50 $\mathrm{mM}$ ammonium bicarbonate buffer for reduction and alkylation. DTT was added to samples to a final concentration of $5 \mathrm{mM}$ for the reduction step, and samples were incubated for $30 \mathrm{~min}$ at $55^{\circ} \mathrm{C}$. After samples were cooled to room temperature, iodoacetomide solution was added to each sample to a final concentration of $15 \mathrm{mM}$. Samples were incubated in the dark for $1 \mathrm{~h}$ at room temperature. Tryptic digestion was performed with $1 \mu \mathrm{g}$ of MS-grade TPCK trypsin, which was added to the samples followed by an incubation at $37^{\circ} \mathrm{C}$ overnight. Samples were split into two fractions and lyophilized prior to phosphopeptide enrichment. Phosphopeptide enrichment was performed using PHOS-Select Iron Affinity Gel (Sigma) or Titansphere Phos-TiO (GL Sciences Inc.) kits using manufacturer protocols. Samples were reconstituted in $0.1 \%$ formic acid and subjected to nLC-ESI-MS/MS on a Proxeon EASY-nLC pump in-line with a hybrid linear quadrupole ion trap Orbitrap mass spectrometer, Velos LTQ, as outlined earlier. For protein identification, Proteowizard was used to convert Thermo.RAW files to the.mzXML, and then searched using X!Tandem on the GPM interface ${ }^{52}$. Search parameters specified a parent MS tolerance of $15 \mathrm{ppm}$ and an MS/MS fragment ion tolerance of $0.4 \mathrm{Da}$, with up to two missed cleavages allowed for trypsin. Oxidation of methionine, ubiquitylation of lysine and carbomidomethylation of cystine residues, phosphorylation of serine and threonine were allowed as variable modifications.

Proximity ligation assay (PLA). Cell lines grown in suspension were centrifuged at $200 \times g$ for $5 \mathrm{~min}$ at room temperature, washed in cold PBS with $2 \% \mathrm{FBS}$, centrifuged at $200 \times \mathrm{g}$ for $5 \mathrm{~min}$ at $4{ }^{\circ} \mathrm{C}$, and finally resuspended in $100 \mu \mathrm{L}$ PBS with $2 \%$ FBS. Cells were then centrifuged onto Superfrost ${ }^{\circledR}$ Plus Micro Slides (48311703, VWR) using the Thermo Scientific ${ }^{\mathrm{m}}$ Cytospin ${ }^{\mathrm{wn}} 4$ Cytocentrifuge at $600 \mathrm{rpm}$ for $10 \mathrm{~min}$. For adherent cell lines, cells were grown on coverslips in 12-well plates. PLA was performed with antibodies against MYC (06-340, Millipore) and/or PNUTS (611060, BD Biosciences) as described previously ${ }^{20}$.

\section{Chromatin immunoprecipitation (ChIP) and ChIP-re-ChIP. ChIP- $q P C R$. ChIP} was performed as described previously ${ }^{53}$ and as follows. ChIP-qPCR for MYC (homemade N262) and PNUTS (A300-440A, Bethyl Laboratories) was performed with $10 \times 10^{6}$ cells and for histone $\mathrm{H} 3$ antibody (ab1791, Abcam) was performed with $5 \times 10^{6}$ cells crosslinked in $1 \%$ formaldehyde per ChIP reaction. Following lysis in Cell Lysis Buffer (1\% SDS, $10 \mathrm{mM}$ EDTA, $50 \mathrm{mM}$ Tris-HCl pH 8.1), samples were sonicated with Bioruptor Pico (Diagenode) and diluted in Dilution Buffer (1\% Triton X-100, 2 mM EDTA, 150 mM NaCl, 20 mM Tris-HCl pH 8.1) with pre-incubated mixture of antibody and Dynabeads (Invitrogen). After overnight incubation, samples were washed in Washing Buffer (50 mM HEPES pH 7.6, $1 \mathrm{mM}$ EDTA, $0.7 \%$ sodium deoxycholate, $1 \% \mathrm{NP}-40,0.5 \mathrm{M} \mathrm{LiCl}$ ) then TE Buffer. DNA was eluted in Decrosslinking Buffer (1\% SDS, $\left.0.1 \mathrm{M} \mathrm{NaHCO}_{3}\right)$ overnight, purified with QIAquick PCR Purification Kit (Qiagen), and analyzed by qPCR. Species matched IgG (Santa Cruz) was used as control for all ChIP experiments.

ChIP-re-ChIP was performed with $40 \times 10^{6}$ cells per reaction. The first IP using the MYC antibody was described above. After washes, chromatin was released using Release Buffer (TE pH 7.5, 1\% SDS, $10 \mathrm{mM} \mathrm{DTT}$ ) at $37^{\circ} \mathrm{C}$ for $30 \mathrm{~min}$ and diluted in Dilution Buffer with pre-incubated mixture of Dynabeads and MAX (73C5a, Abcam) or PNUTS antibodies or IgG. Following washes and decrosslinking, DNA was purified and analyzed by qPCR.

Immunoprecipitation. Cell lysate was incubated with MYC (9E10, homemade) or IgG (sc-2027, Santa Cruz Biotechnology) antibodies with $15 \mu \mathrm{L}$ protein Gsepharose beads (GE Healthcare) overnight to end-over-end rotation at $4{ }^{\circ} \mathrm{C}$. Next day, beads were pelleted $\left(425 \times g\right.$ for $2 \mathrm{~min}$ at $\left.4^{\circ} \mathrm{C}\right)$ and washed three times with $0.1 \%$ NP40 in PBS. The IP beads were resuspended in suitable buffers as described.
2D electrophoresis and immunoblotting. MYC was immunoprecipitated as described above and the MYC IP beads then resuspended in rehydration buffer $(8$ M Urea, 2\% CHAPS, 25 mM DTT, 20 pg/mL IPG buffer pH 4-7 (17-6000-86, GE Healthcare), bromophenol blue), centrifuged and $125 \mu \mathrm{L}$ of supernatant incubated with immobiline drystrip pH 4-7, $7 \mathrm{~cm}$ (17-6001-10, GE Healthcare). The IPG strip was covered with drystrip cover fluid (17-1335-01, GE healthcare) overnight in a reswelling tray. Next day, IPG strips were focused on Ettan IPGphor 3 (GE healthcare) at $300 \mathrm{~V}$ for $30 \mathrm{~min}, 300-10,000 \mathrm{~V}$ for $3 \mathrm{~h}$, and $10,000 \mathrm{~V}$ for $4 \mathrm{~h}$. The IPG strips were then washed with equilibration buffer I ( $6 \mathrm{M}$ Urea, $0.375 \mathrm{M}$ Tris pH 8.8, $2 \%$ SDS, $20 \%$ glycerol, and $20 \mathrm{mg} / \mathrm{mL}$ DTT) and equilibration buffer II (6 M Urea, $0.375 \mathrm{M}$ Tris pH 8.8, $2 \%$ SDS, $20 \%$ glycerol, and $25 \mathrm{mg} / \mathrm{mL}$ Iodoacetamide) for $10 \mathrm{~min}$ on shaker. The IPG strips were then rinsed with SDS PAGE running buffer and overlaid onto a $10 \%$ SDS-PAGE with $0.5 \%$ agarose. The SDSPAGE and immunoblotting was conducted as described earlier ${ }^{54}$.

Phosphatase treatment. MYC was immunoprecipitated as described above and then the MYC IP beads were treated with $12.5 \mathrm{U}$ protein phosphatase 1 (P07545, New England BioLabs) in NEB buffer and $\mathrm{MnCl}_{2}$ or $12.5 \mathrm{U}$ CIP (M0290L, New England BioLabs) in NEB buffer 3 for $2 \mathrm{~h}$ at $30^{\circ} \mathrm{C}$. The beads were washed once with $0.1 \%$ NP40 in PBS, eluted with $2 \mathrm{X}$ sample buffer, incubated at $95^{\circ} \mathrm{C}$ for $5 \mathrm{~min}$ and loaded onto a $10 \%$ SDS-PAGE. To measure enzyme activity $1 \mu \mathrm{L}$ of the CIP or $5 \mu \mathrm{L}$ of the PP1 sample was removed at the end of the $2 \mathrm{~h}$ incubation period and prior to bead washing. These samples were then incubated with the substrate 50 mM PNP (P0757, New England BioLabs) in the above mentioned buffer for CIP or PP1 for $5 \mathrm{~min}$ or $30 \mathrm{~min}$ respectively at $30^{\circ} \mathrm{C}$ in a $50 \mu \mathrm{L}$ reaction volume. The reaction was stopped with $1 \mathrm{~mL} 1 \mathrm{~N} \mathrm{NaOH}$ and the amount of $p$-Nitrophenol formed was determined by spectrophotometer absorbance at $405 \mathrm{nM}$ (molar extinction coefficient $18,000 \mathrm{M}-1 \mathrm{~cm}-1$ ).

Cellular fractionation. Cells grown in a tissue culture dish were washed once with PBS, scraped, collected into eppendorf tubes, and centrifuged at $1400 \mathrm{rpm}$ for 2 min at $4{ }^{\circ} \mathrm{C}$. Cell pellets were then resuspended in buffer A $(10 \mathrm{mM}$ HEPES $\mathrm{pH} 7.9$, $10 \mathrm{mM} \mathrm{KCl}, 0.1 \mathrm{mM}$ EDTA, $0.1 \mathrm{mM}$ EGTA, $1 \mathrm{mM}$ DTT, protease inhibitor cocktail (P8340, Sigma-Millipore), incubated on ice for $15 \mathrm{~min}$, vortexed, and centrifuged at $3000 \mathrm{rpm}$ for $5 \mathrm{~min}$ at $4{ }^{\circ} \mathrm{C}$. The supernatant (cytoplasmic fraction) was collected in an eppendorf tube and pellet (nuclear fraction) was washed once with buffer $\mathrm{A}$, centrifuged at $3000 \mathrm{rpm}$ for $5 \mathrm{~min}$ at $4{ }^{\circ} \mathrm{C}$. The pellet was lysed in SDS lysis buffer $(0.05 \mathrm{M}$ Tris pH 6.8, $1 \%$ SDS, $10 \% \beta$-mercaptoethanol, and $10 \%$ glycerol) and passed through $25 \mathrm{G}$ needle syringe a few times. The cytoplasmic fraction was also mixed with $4 \times$ SDS lysis buffer. The nuclear and cytoplasmic extracts were heated at $95^{\circ} \mathrm{C}$ for $5 \mathrm{~min}$ and loaded onto an SDS-PAGE.

Antibodies, primers, and siRNAs. Antibodies for MYC pT58 (04-217, Millipore, used at 1:2000), MYC p62 (ab78318, abcam, used at 1:1000), MAX for ChIP (sc765, Santa Cruz, $10 \mu \mathrm{g}$ used for ChIP), PP1a isoform (438100, Invitrogen, used at $1: 2000)$, PP1 $\beta$ isoform (ab53315, abcam, used at 1:1000), PP1 $\gamma$ isoform (A300906A, Bethyl laboratories, used at 1:5000), biotin anti-mouse SV40 large T and small $\mathrm{T}$ antigen (554151, BD Biosciences, used at 1:1000), PNUTS for immunoblotting (A300-439A, Bethyl Laboratories, used at 1:15,000 to 1:20,000), MYC for ChIP (homemade N262, $2 \mu \mathrm{g}$ used for ChIP, $4 \mu \mathrm{g}$ used for re-ChIP), PNUTS for ChIP (A300-440A, Bethyl Laboratories, $4 \mu \mathrm{g}$ for ChIP, $10 \mu \mathrm{g}$ for re-ChIP), Actin (A2066, Sigma, used at 1:10000), Anti-mouse HRP (NA931V, GE healthcare, used at 1:10,000), Anti-tubulin (DM1A, Calbiochem, used at 1:2000), Anti-laminB1 (ab16048, Abcam, used at 1:1000) and Anti-rabbit HRP (NA934V, Sigma, used at $1: 10,000)$.

The qPCR primers for PP1 $\alpha$ isoform (fwd GTTCCTCCACAAGCACGACT, rev GTTCCTCCACAAGCACGACT), PP $1 \beta$ isoform (fwd GAAGATCTTCTGTTGTC ATG, rev GCACATCCTTATCTGGATCAGAC), PP1 $\gamma$ isoform (fwd ACTAGAAC TTGAAGCACCACT, rev CGCAGCAAATCATAGTATTGTCC), and RPLPO (fwd CAGATTGGCTACCCAACTGTT, rev GGGAAGGTGTAATCCGTCTCC). The qPCR primers for ChIP for CAD (fwd ACGTGGACCGACTCCGG, rev CCA TGGGAAGGGAACTCAGA), CDK4 (fwd AGGCATGTGTCATGTGTGATCTT, rev CCGCTCCCAGTCTTCCTTG, HES1 (fwd AATGAGATCCGGAATCGGCG, rev TCATCCGTAGGCTTTAGGTTCTG), LDHA (fwd ACGTCAGCATAGCTG TTCCA, rev AATGAGATCCGGAATCGGCG) NCL (fwd TTGCGACGCGTACG AGCTGG, rev ACTCCGACTAGGGCCGATAC), PPP1R10 (fwd TGCTAGTGAA ACGCCCTTGT, rev GCCAATCAGTTGGCGAGTTG), Chr6 (fwd TGGCATTGT CCTAATACTTCAGTGAT, rev TTTCTGAAGTGCTGCTACCTCTCA)

The protein phosphatase catalytic subunit siRNAs were from OriGene; PPP1CA- SR303671A (GAGACGCUACAACAUCAAACUGUGG) and SR303671B (AGACGGCUACGAGUUCUUUGCCAAG), PPP1CB- SR303672A (CUAAUAGAAAGAUGUGCUACACUGT) and SR303672B (GCUUUGUAGUGAAGUAUAGUAGCAA), PPP1CC- SR303673A (GCUUCAGGAGAAUGAAAUCAGAGGA) and SR303673B (CGAUGGCGGAUUUAGAUAAACUCAA).

Co-amplification heatmap. The occurrence of co-amplification of $M Y C$ with PPP1R10, PPP1CA, PPP1CB, and PPP1CC was assessed in multiple cancer types using datasets provided by The Cancer Genome Atlas (TCGA). DNA genome-wide 
somatic copy number and clinical profiles were downloaded from Broad GDAC Firehouse (https://gdac.broadinstitute.org/), release 2014-10-17. GISTIC v223 level 4 data was used for somatic copy-number analysis and all data pre-processing was completed using the $\mathrm{R}$ statistical environment (v.3.1.3). The Chi-square test of independence was applied to test the dependence of PPP1R10 amplification, along with PP1 subunits, on MYC amplification using the R statistical environment (v.3.2.5). All visualizations were completed using lattice (v.0.20-33) and latticeExtra (v.0.6-28).

Statistical analysis. All the graphs show median with range or mean with error bars representing s.d. of a minimum of three biological replicates. Differences in groups for bar graphs were analyzed using one-way ANOVA with Bonferroni test or for grouped bar graphs using two-way ANOVA with Bonferroni test using Graphpad Prism Software. For BioID data, proteins identified with a cut-off of 0.8 using ProteinProphet (corresponding to FDR $<1 \%$ ) were analyzed with SAINT Express v. $3.3^{50,51}$

Data availability. The BioID and PTM mass spectrometry datasets generated and analysed during the current study are available in the MassIVE repository (accession number: MSV000082424). The data that support the findings of this study are available from the corresponding author upon request.

Received: 23 November 2016 Accepted: 6 July 2018

Published online: 29 August 2018

\section{References}

1. Farrell A. S., \& SearsR. C. MYC degradation.Cold Spring Harb. Perspect. Med. 4, a014365 (2014).

2. Meyer, N. \& Penn, L. Z. Reflecting on 25 years with MYC. Nat. Rev. Cancer 8, 976-990 (2008).

3. Conacci-Sorrell, M., McFerrin, L. \& Eisenman, R. N. An overview of MYC and its interactome. Cold Spring Harb. Perspect. Med. 4, a014357 (2014).

4. Dang, C. V. MYC on the path to cancer. Cell 149, 22-35 (2012).

5. Stine, Z. E., Walton, Z. E., Altman, B. J., Hsieh, A. L. \& Dang, C. V. MYC, metabolism, and cancer. Cancer Discov. 5, 1024-1039 (2015).

6. Gabay, M., Li, Y., \& Felsher, D. W. MYC activation is a hallmark of cancer initiation and maintenance. Cold Spring Harb. Perspect. Med. 4, a014241 (2014).

7. Wahlstrom, T. \& Henriksson, M. A. Impact of MYC in regulation of tumor cell metabolism. Biochim. Biophys. Acta 1849, 563-569 (2015).

8. Kress, T. R., Sabo, A. \& Amati, B. MYC: connecting selective transcriptional control to global RNA production. Nat. Rev. Cancer 15, 593-607 (2015).

9. Wolf, E., Lin, C. Y., Eilers, M. \& Levens, D. L. Taming of the beast: shaping Myc-dependent amplification. Trends Cell Biol. 25, 241-248 (2015).

10. Luscher, B. \& Vervoorts, J. Regulation of gene transcription by the oncoprotein MYC. Gene 494, 145-160 (2012).

11. Vervoorts, J., Luscher-Firzlaff, J. \& Luscher, B. The ins and outs of MYC regulation by posttranslational mechanisms. J. Biol. Chem. 281, 34725-34729 (2006).

12. Soucek, L. et al. Modelling Myc inhibition as a cancer therapy. Nature 455, 679-683 (2008).

13. Soucek, L. et al. Inhibition of Myc family proteins eradicates KRas-driven lung cancer in mice. Genes Dev. 27, 504-513 (2013).

14. Fletcher, S. \& Prochownik, E. V. Small-molecule inhibitors of the Myc oncoprotein. Biochim. Biophys. Acta 1849, 525-543 (2015).

15. Albihn, A., Johnsen, J. I. \& Henriksson, M. A. MYC in oncogenesis and as a target for cancer therapies. Adv. Cancer Res. 107, 163-224 (2010).

16. Zuber, J. et al. RNAi screen identifies Brd4 as a therapeutic target in acute myeloid leukaemia. Nature 478, 524-528 (2011).

17. Delmore, J. E. et al. BET bromodomain inhibition as a therapeutic strategy to target c-Myc. Cell 146, 904-917 (2011)

18. Amorim, S. et al. Bromodomain inhibitor OTX015 in patients with lymphoma or multiple myeloma: a dose-escalation, open-label, pharmacokinetic, phase 1 study. Lancet Haematol. 3, e196-e204 (2016).

19. Wasylishen, A. R. et al. MYC phosphorylation at novel regulatory regions suppresses transforming activity. Cancer Res. 73, 6504-15 (2013)

20. Dingar, D. et al. BioID identifies novel c-MYC interacting partners in cultured cells and xenograft tumors. J. Proteom. 118, 95-111 (2015).

21. Roux, K. J., Kim, D. I., Raida, M. \& Burke, B. A promiscuous biotin ligase fusion protein identifies proximal and interacting proteins in mammalian cells. J. Cell Biol. 196, 801-810 (2012).

22. Kalkat, M., Wasylishen, A. R., Kim, S. S. \& Penn, L. More than MAX: discovering the Myc interactome. Cell Cycle 10, 374-375 (2011).
23. Blackwood, E. M. \& Eisenman, R. N. Max: a helix-loop-helix zipper protein that forms a sequence-specific DNA-binding complex with Myc. Science 251, 1211-1217 (1991)

24. McMahon, S. B., Van Buskirk, H. A., Dugan, K. A., Copeland, T. D. \& Cole, M. D. The novel ATM-related protein TRRAP is an essential cofactor for the c-Myc and E2F oncoproteins. Cell 94, 363-374 (1998).

25. Thomas, L. R. et al. Interaction with WDR5 promotes target gene recognition and tumorigenesis by MYC. Mol. Cell 58, 440-452 (2015).

26. Helander, S. et al. Pre-anchoring of Pin1 to unphosphorylated c-Myc in a fuzzy complex regulates c-Myc activity. Structure 23, 2267-2279 (2015).

27. Hann, S. R. Role of post-translational modifications in regulating c-Myc proteolysis, transcriptional activity and biological function. Semin. Cancer Biol. 16, 288-302 (2006).

28. Huang, Z., Traugh, J. A. \& Bishop, J. M. Negative control of the Myc protein by the stress-responsive kinase Pak2. Mol. Cell Biol. 24, 1582-1594 (2004).

29. Chakraborty, A. A. et al. A common functional consequence of tumor-derived mutations within c-MYC. Oncogene 34, 2406-2409 (2015).

30. Hemann, M. T. et al. Evasion of the p53 tumour surveillance network by tumour-derived MYC mutants. Nature 436, 807-811 (2005).

31. Rebelo, S., Santos, M., Martins, F., da Cruz e Silva, E. F. \& da Cruz e Silva, O A. Protein phosphatase 1 is a key player in nuclear events. Cell Signal. 27, 2589-2598 (2015).

32. Choy, M. S. et al. Understanding the antagonism of retinoblastoma protein dephosphorylation by PNUTS provides insights into the PP1 regulatory code. Proc. Natl Acad. Sci. USA 111, 4097-4102 (2014).

33. Ciurciu, A. et al. PNUTS/PP1 regulates RNAPII-mediated gene expression and is necessary for developmental growth. PLoS Genet. 9, e1003885 (2013)

34. von der Lehr, N. et al. The F-box protein Skp2 participates in c-Myc proteosomal degradation and acts as a cofactor for c-Myc-regulated transcription. Mol. Cell 11, 1189-1200 (2003).

35. Bahram, F., von der Lehr, N., Cetinkaya, C. \& Larsson, L. G. c-Myc hot spot mutations in lymphomas result in inefficient ubiquitination and decreased proteasome-mediated turnover. Blood 95, 2104-2110 (2000).

36. Resjo, S., Oknianska, A., Zolnierowicz, S., Manganiello, V. \& Degerman, E. Phosphorylation and activation of phosphodiesterase type 3B (PDE3B) in adipocytes in response to serine/threonine phosphatase inhibitors: deactivation of PDE3B in vitro by protein phosphatase type 2A. Biochem. J. 341, 839-845 (1999).

37. Yeh, E. et al. A signalling pathway controlling c-Myc degradation that impacts oncogenic transformation of human cells. Nat. Cell Biol. 6, 308-318 (2004).

38. Rajagopalan, H. et al. Inactivation of hCDC4 can cause chromosomal instability. Nature 428, 77-81 (2004).

39. Verheyen, T. et al. Genome-wide promoter binding profiling of protein phosphatase-1 and its major nuclear targeting subunits. Nucleic Acids Res. 43, 5771-5784 (2015)

40. Xu, J. et al. Eya1 interacts with Six 2 and Myc to regulate expansion of the nephron progenitor pool during nephrogenesis. Dev. Cell 31, 434-447 (2014).

41. Thomas, L. R. \& Tansey, W. P. Proteolytic control of the oncoprotein transcription factor Myc. Adv. Cancer Res. 110, 77-106 (2011).

42. Kim, J. Y. et al. c-Myc phosphorylation by PKCzeta represses prostate tumorigenesis. Proc. Natl Acad. Sci. USA 110, 6418-6423 (2013).

43. Luscher, B. \& Eisenman, R. N. Mitosis-specific phosphorylation of the nuclear oncoproteins Myc and Myb. J. Cell Biol. 118, 775-784 (1992).

44. Jerebtsova, M. et al. Mass spectrometry and biochemical analysis of RNA polymerase II: targeting by protein phosphatase-1. Mol. Cell Biochem. 347, 79-87 (2011).

45. Debnath, J., Muthuswamy, S. K. \& Brugge, J. S. Morphogenesis and oncogenesis of MCF-10A mammary epithelial acini grown in threedimensional basement membrane cultures. Methods 30, 256-268 (2003).

46. Kessner, D., Chambers, M., Burke, R., Agus, D. \& Mallick, P. ProteoWizard: open source software for rapid proteomics tools development. Bioinformatics 24, 2534-2536 (2008).

47. Craig, R. \& Beavis, R. C. TANDEM: matching proteins with tandem mass spectra. Bioinformatics 20, 1466-1467 (2004).

48. Pedrioli, P. G. Trans-proteomic pipeline: a pipeline for proteomic analysis Methods Mol. Biol. 604, 213-238 (2010).

49. Liu, G. et al. ProHits: integrated software for mass spectrometry-based interaction proteomics. Nat. Biotechnol. 28, 1015-1017 (2010).

50. Choi, H. et al. SAINT: probabilistic scoring of affinity purification-mass spectrometry data. Nat. Methods 8, 70-73 (2011).

51. Teo, G. et al. SAINTexpress: improvements and additional features in significance analysis of INTeractome software. J. Proteom. 100, 37-43 (2014)

52. Fenyo, D. \& Beavis, R. C. The GPMDB REST interface. Bioinformatics 31, 2056-2058 (2015)

53. Stojanova, A. et al. MYC interaction with the tumor suppressive SWI/SNF complex member INI1 regulates transcription and cellular transformation. Cell Cycle 15, 1693-1705 (2016). 
54. Dingar, D. et al. Effect of pressure overload-induced hypertrophy on the expression and localization of p38 MAP kinase isoforms in the mouse heart. Cell Signal. 22, 1634-1644 (2010).

\section{Acknowledgements}

Special thanks to Melania Pintilie for generously sharing her time and providing expert advice regarding biostatistical data analysis and to Dr. Rosalie C. Sears for providing Small T antigen for this work. D.D. holds a Princess Margaret Cancer Centre Knudson Postdoctoral Fellowship. D.R. holds a Natural Sciences and Engineering Research Council of Canada. M.K. and W.B.T. hold Canadian Breast Cancer Research Ontario Region Doctoral Fellowships. L.Z.P holds the Canada Research Chair (CRC) in Molecular Oncology, and B.R. holds the CRC in Proteomics and Molecular Medicine. Funding in the L.Z.P. and B.R. labs were provided in by a Canadian Institutes of Health Research (CIHR) project grant PJT156167 and operating grant MOP130340, respectively. P.C.B. was supported by a CIHR New Investigator Award and TFRI New Investigator Award. The results described here are in part based upon data generated by the TCGA Research Network: http://cancergenome.nih.gov/.

\section{Author contributions}

D.D. and L.Z.P. wrote and co-authors edited the manuscript; L.Z.P. supervised the entire project; D.D. designed and performed immunoblotting, IP, PLA, 2D, nuclear and cytoplasmic fractionation, ENCODE analysis; D.D. designed and W.B.T. performed and analysed ChIP experiments; M.K. D.R., P.K.C., and B.R. performed and analysed MS; C. L. performed cyclohexamide experiments. C.L. and J.D. performed cloning and immunoblotting; A.T. performed PLA, K.E.H. and P.C.B. analysed patient gene amplification data.

\section{Additional information}

Supplementary Information accompanies this paper at https://doi.org/10.1038/s41467018-05660-0.

Competing interests: The authors declare no competing interests.

Reprints and permission information is available online at http://npg.nature.com/ reprintsandpermissions/

Publisher's note: Springer Nature remains neutral with regard to jurisdictional claims in published maps and institutional affiliations.

(c) Open Access This article is licensed under a Creative Commons Attribution 4.0 International License, which permits use, sharing, adaptation, distribution and reproduction in any medium or format, as long as you give appropriate credit to the original author(s) and the source, provide a link to the Creative Commons license, and indicate if changes were made. The images or other third party material in this article are included in the article's Creative Commons license, unless indicated otherwise in a credit line to the material. If material is not included in the article's Creative Commons license and your intended use is not permitted by statutory regulation or exceeds the permitted use, you will need to obtain permission directly from the copyright holder. To view a copy of this license, visit http://creativecommons.org/ licenses/by/4.0/.

(C) The Author(s) 2018 